\title{
Modellierung der Strömungsdynamik in einem revitalisierten Küstenmoorgebiet an der Ostsee
}

\author{
Lisa Schreiber ${ }^{1,2} \cdot$ Matthias Munz $^{1} \cdot$ Thomas Salzmann $^{3} \cdot$ Sascha E. Oswald ${ }^{1}$ \\ Eingegangen: 16. Dezember 2020 / Überarbeitet: 17. Februar 2021 / Angenommen: 4. Mai 2021 / Online publiziert: 14. Juni 2021 \\ (c) Der/die Autor(en) 2021
}

\section{Zusammenfassung}

Küstennahe Niedermoore wurden durch den Menschen verändert, bspw. durch das Anlegen von Entwässerungsgräben, dem Bau von Küstenschutzdeichen oder aktuell einer Renaturierung. Außerdem ist es wichtig die komplexe Interaktion mit der See zu verstehen, um Aussagen über die zukünftige Entwicklung treffen zu können. In der vorliegenden Studie wurde die ober- und unterirdische Strömung in einem Feuchtgebiet an der mecklenburgischen Ostseeküste nahe Warnemünde (Deutschland) untersucht, um dessen wechselseitigen Austausch mit der Ostsee zu quantifizieren und zu analysieren, wie sich ein Sturmhochwasserereignis auf den Salzeintrag ins Gebiet auswirkt. Hierfür wurde ein dreidimensionales instationäres Grundwassermodell erstellt, mit einem eindimensionalen Modell des Grabensystems gekoppelt und mit Messungen im Gebiet kalibriert und verglichen. Die Ergebnisse zeigen, dass neben der oberirdischen Entwässerung auch der Grundwasserabstrom in Richtung Ostsee eine wesentliche Komponente der Wasserbilanz darstellt. Das Verhalten entlang der Küste wird deutlich durch die Dynamik der Ostseewasserstände geprägt, wobei ein Grundwasserabstrom mit einem Zustrom von Ostseewasser bei hohen Küstenwasserständen innerhalb täglicher bis wöchentlicher Zeitskalen wechselt.

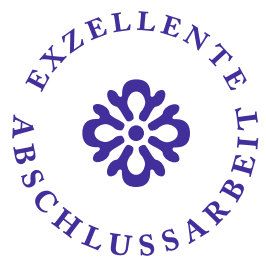

Schlüsselwörter Numerische Modellierung · Ostseeküste · Grundwasser-Oberflächenwasser-Interaktion ·

Sturmhochwasser · Versalzung

\section{Coupled simulation of groundwater and drainage dynamics in a coastal fen}

\section{Abstract}

Coastal wetlands are characterized by continued human influence, e.g. with drainage ditches, coastal dikes or landscape restoration. In addition, it is important to understand the complex interactions with the sea to predict impacts of further development. In the present study the aim was to analyze surface and subsurface flow in a coastal wetland located at the Baltic Sea coastline near Warnemünde (Germany) to quantify water exchange with the Baltic Sea and analyze the effect of a storm flood event on saline intrusion. A 3-D transient groundwater model and a one-dimensional surface water model were set up and calibrated by using hydraulic head measurements. The results indicate that in addition to ditch flow, groundwater discharge to the Baltic Sea often has a significant influence on the overall water budget of the fen. From the transient modelling it became evident that water exchange between groundwater in the fen and the Baltic

Sascha E. Oswald

sascha.oswald@uni-potsdam.de

1 Institut für Umweltwissenschaften und Geographie, Universität Potsdam, Karl-Liebknecht-Str.

24-25, 14476 Potsdam, Deutschland
2 HYDOR Consult GmbH, Am Borsigturm 31, 13507 Berlin, Deutschland

3 Hydrologie und Angewandte Meteorologie, Agrar- und Umweltwissenschaftliche Fakultät, Universität Rostock, Satower Straße 48, 18059 Rostock, Deutschland 
Sea depends on sea level and very often fluctuates between seaward and landward flow directions on daily to weekly time scales.

Keywords Numerical modeling $\cdot$ Baltic Sea coast $\cdot$ Groundwater-surface water interaction $\cdot$ Storm flood $\cdot$ Salinization

\section{Einführung}

Feuchtgebiete, wie etwa Moore, sind komplexe Ökosysteme und spielen u. a. für die Biodiversität, die Wasserqualität, den Wasserhaushalt sowie den Natur- und Klimaschutz eine bedeutende Rolle. Naturnahe Moore stellen Ökosystemdienstleistungen bereit, wozu bspw. die Dämpfung von Abflussspitzen und die Filterung von Wasserinhaltsstoffen gehören. Sie senken den Nährstoffeintrag in umliegende Oberflächengewässer und sind Wasserspeicher für Grundund Oberflächenwasser (LLUR SH 2012). Die anthropogen bedingte Veränderung von Feuchtgebieten, z.B. durch das Anlegen von Entwässerungsgräben, geht i.d.R. mit einer starken Modifikation des hydrologischen Prozessgeschehens einher (Thompson et al. 2004). In Deutschland sind aufgrund von Entwässerungen über $95 \%$ der Moore von Torfzersetzung betroffen (LLUR SH 2012). Eine Renaturierung dieser Areale ist oftmals nur durch neuerliche Eingriffe in das hydrologische System möglich, wie bspw. durch Verfüllen der Entwässerungsgräben oder Wiedervernässung durch Staubauwerke (Thompson et al. 2004). Teilweise wird in küstennahen Mooren die Unterhaltung und Pflege der Dünen eingestellt, sodass es in Zusammenspiel mit dem Anstieg des Meeresspiegels zunehmend zum oberund unterirdischen Eintrag von Salzwasser bei Sturmhochwassern kommen kann.

Die hydro(geo)logischen Prozesse innerhalb von Feuchtgebieten sind sehr komplex. So sind Feuchtgebiete durch eine starke Interaktion von Grund- und Oberflächenwasser gekennzeichnet (Rossi et al. 2012). Bei küstennahen Feuchtgebieten kann der Austausch von Wasser sowie Substanzen über die Grenzfläche zum Meer eine wichtige Komponente im Wasser- und Stoffhaushalt darstellen $(\mathrm{Qu}$ et al. 2017). Dies betrifft u. a. Meeressalz auf der einen Seite, Nähr- und organische Schadstoffe auf der anderen. Die Wasser- und Stoffflüsse in küstennahen Feuchtgebieten finden auf verschiedenen räumlichen sowie zeitlichen Skalen statt und sind daher schwer zu quantifizieren. Einzelereignisse wie meerseitige Überflutungen durch Sturmhochwasser können einen substanziellen oder sogar langanhaltenden Einfluss ausüben, insbesondere hinsichtlich einer Versalzung.

Für ein besseres Prozessverständnis und zur Quantifizierung der einzelnen Wasserhaushaltskomponenten können hydrogeologische Modelle in Kombination mit Modellen für Strömung in Oberflächengewässern genutzt werden. Langevin et al. (2005) simulierten die Wasserströmung sowie die Salinität in einem küstennahen Feuchtgebiet in der Umgebung der Everglades, Florida, mithilfe eines gekoppelten, dreidimensionalen, dichteabhängigen Oberflächenwasser-Grundwassermodells (SWIFT2D-SEAWAT). Yang et al. (2013) simulierten die Auswirkungen von Tiden und Sturmfluten auf die Strömungen in küstennahen Bereichen unter Berücksichtigung der Dichteabhängigkeit mithilfe von HydroGeoSphere. Außerdem nutzten Monninkhoff und Li (2009) eine Kopplung von FEFLOW mit MIKE 11 für die Optimierung der Überflutungsstrategie und zur Abschätzung des Grundwassereinflusses im Bereich der Unteren Havel. Später wurde mit einer solchen Modellkopplung der Flutungsprozess eines Tagebaurestsees (Cottbuser Ostsee) und die resultierenden Auswirkungen auf die umgebenden Flächen simuliert (Monninkhoff et al. 2014).

Das Ziel unserer Studie ist es, mittels gekoppelter 3DSimulationen die Grundwasserströmungsdynamik des küstennahen Niedermoores „Hütelmoor und Heiligensee“ und dessen wechselseitigen Austausch mit der Ostsee zu erfassen und zu quantifizieren. Durch eine Simulation des Sturmhochwassers der Ostsee am 4./5. Januar 2017 sollen die relevanten Prozesse der Salzwasserintrusion und -verteilung im Untersuchungsgebiet erfasst und abgebildet werden. Die Simulationen sollen damit vor allem dazu beitragen, Wasserflüsse genauer zu quantifizieren und die Vorstellung über das Prozessgeschehen in solch einem Untersuchungsgebiet weiter zu schärfen.

\section{Untersuchungsgebiet}

Das ca. 490ha große Untersuchungsgebiet liegt an der mecklenburgischen Ostseeküste, östlich von Warnemünde, Deutschland (Abb. 1). Es umfasst zu einem großen Teil das Naturschutzgebiet „Heiligensee und Hütelmoor“ (NSG). Der Schutzzweck des NSGs besteht in der Erhaltung des küstennahen Versumpfungsmoores (Umweltministerium M-V 2003). In diesem Gebiet wurden in den letzten 20 Jahren bereits umfangreiche Beobachtungsdaten erhoben und ausgewertet. Dahms (1991) hat die Wasserstände im Grundwasser sowie im Grabensystem untersucht und verschiedene Varianten für die Nutzung der im Gebiet vorhandenen Wasserregulierungseinrichtungen, wie ein bis 1993 betriebenes Schöpfwerk zur Entwässerung des Niedermoores, aufgestellt. Krüger (1995) kartierte nach Stilllegung des Schöpfwerkes die elektrischen Leitfähig- 


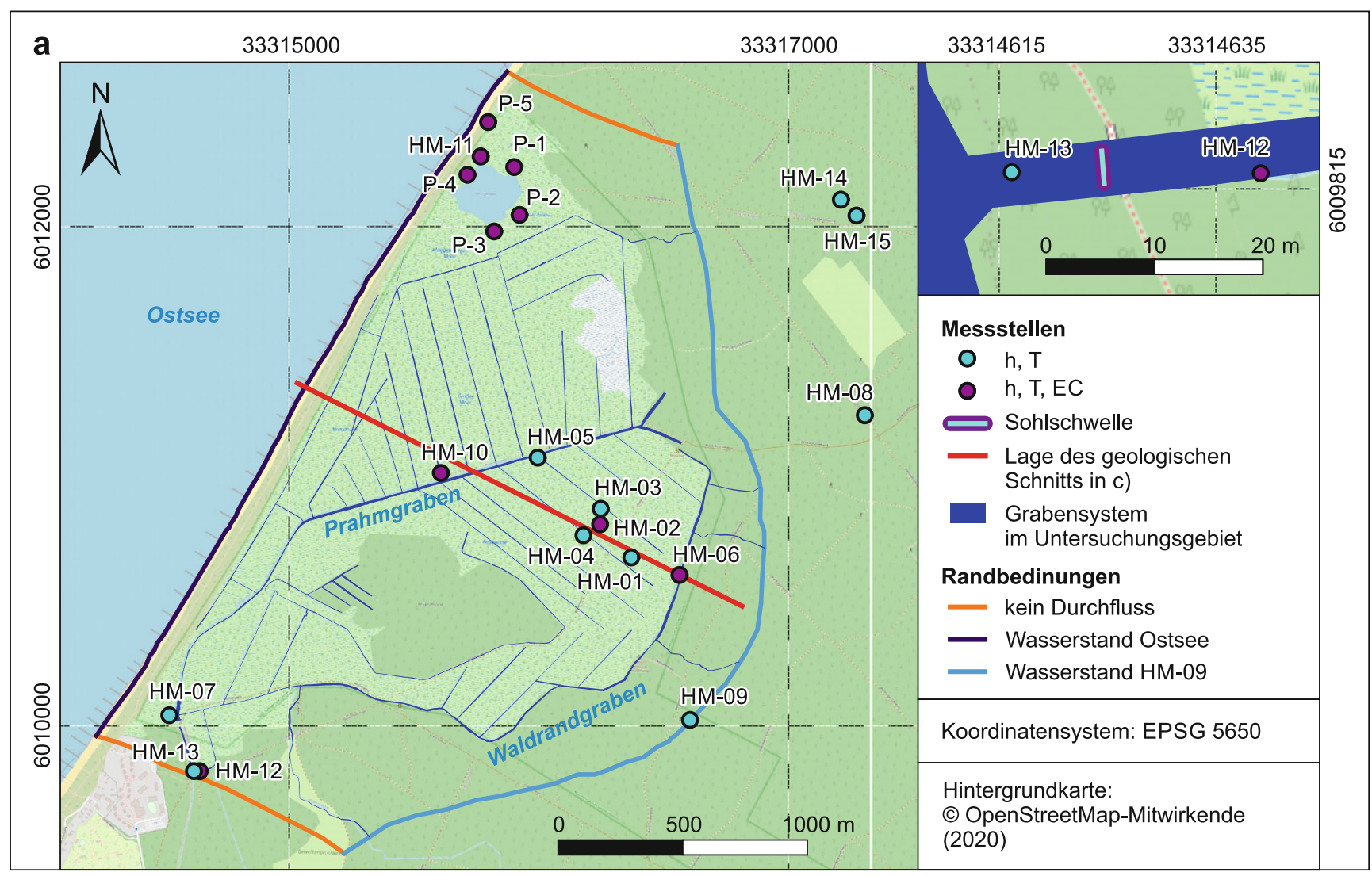

Abb. 1 a Untersuchungsgebiet inklusive der Oberflächenwasser- und Grundwassermessstellen für die Erfassung des Wasserstandes (h), der Temperatur (T) und der elektrischen Leitfähigkeit (EC) sowie Darstellung der Randbedingungen. Das Detail oben rechts verdeutlicht die Lage der Sohlschwelle und der angrenzenden Messstellen am Gebietsauslass im Südwesten.

Fig. 1 a Study area with surface water (purple dots) and groundwater (turquoise dots), observation wells for continuous recording of water level (h), temperature (T) and electrical conductivity (EC). The blue (transient hydraulic head) and the orange (no-flow) lines indicate the inland boundary conditions in FEFLOW. The detail on the top right illustrates the location of the groundsill and the adjacent measuring points at the outlet in the southwest.

keiten (EC) in der Niederung sowie in den umgebenden Waldbereichen. Die aktuelle hydrologische Grundcharakterisierung, eine langjährig gemittelte Betrachtung des heutigen Wasserhaushaltes in der Niederung sowie die hydraulischen Auswirkungen eines Sturmhochwassers auf die Niederung ist in den Studien von Miegel et al. (2016, 2017) und Selle et al. (2016) beschrieben. In diesen Studien wird der unterirdische Austausch zwischen Niederung und Ostsee aufgrund der lokalen Gegebenheiten (Verdichtung des Torfes durch Baumaßnahmen im Bereich der Düne mit resultierenden niedrigen Durchlässigkeitsbeiwerten) als insgesamt gering eingeschätzt. Allerdings wurde auch festgestellt, dass genauere Aussagen über die Grundwasserströmung und zu den örtlichen Verhältnissen im Bereich der Düne nicht ohne eine aufwändige Modellierung möglich sind. Im Gegensatz zu den vorausgegangenen Ergebnissen wurden meerseitig auch potenzielle Grundwasseraustrittsgebiete identifiziert (Jurasinski et al. 2018; Kreuzburg et al. 2018). Aktuell werden hydrodynamische, (bio-)geochemische und biologische Wechselwirkungen zwischen der
Ostsee und der angrenzenden Niederung durch das DFGGraduiertenkolleg „Baltic TRANSCOAST“ der Universität Rostock intensiv untersucht (Jurasinski et al. 2018).

Das Klima im Untersuchungsgebiet liegt im Übergangsbereich des atlantisch-marinen und des kontinentalen Klimas (Krüger 1995) und ist regional stark durch die Ostsee geprägt (Dahms 1991; Miegel et al. 2016). Die durchschnittliche Jahrestemperatur beträgt $9,5^{\circ} \mathrm{C}\left(\min =4,7^{\circ} \mathrm{C}\right.$, $\max =10,4^{\circ} \mathrm{C}$ ), und der durchschnittliche, nach Richter (1995) korrigierte, jährliche Niederschlag $701 \mathrm{~mm}$ $(\min =489 \mathrm{~mm}, \max =1032 \mathrm{~mm}) \quad($ Klimastation RostockWarnemünde, DWD 2018b).

Das Untersuchungsgebiet umfasst eine näherungsweise halbkreisförmige, flache Niederung ( $-0,2$ bis $0,4 \mathrm{~m}$ ü. NHN) mit dem im Nordosten liegenden Heiligensee (Abb. 1a) und wird nordwestlich durch eine langgezogene Düne zur Ostsee begrenzt (Abb. 1b, c). In der nordwestlichen Umgebung des Heiligensees befindet sich eine potenzielle Durchbruchstelle durch die Düne, über die Ostseewasser bei Sturmhochwassern lokal ins Gebiet eindringen kann (Selle et al. 


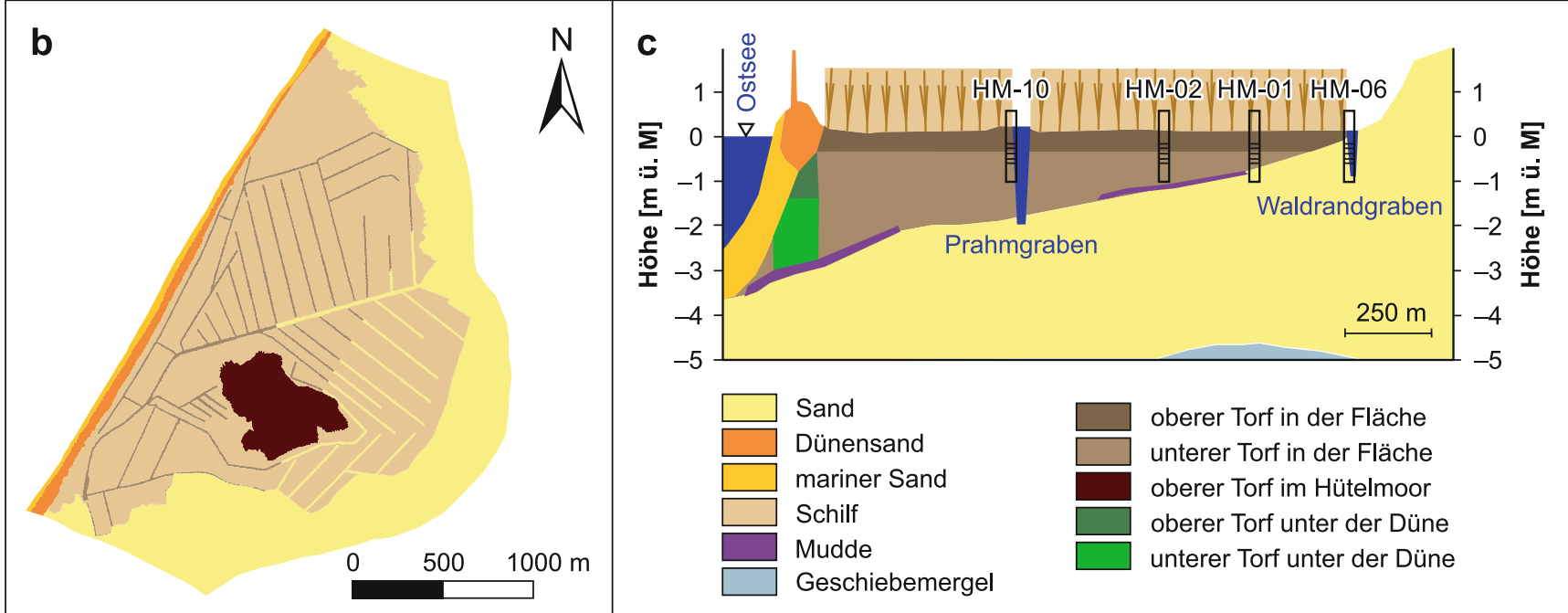

Abb. 1 b Vereinfachte Darstellung der anstehenden hydrogeologischen Modellzonen. c Vereinfachter geologischer Schnitt vom Strand über die Niederung bis an den Waldrand in Richtung Westnordwest-Ostsüdost in der Mitte des Gebiets mit Auftragung ausgewählter Messstellen entlang des Schnittes. (Verändert nach Jurasinski et al. (2018) nach Ibenthal (2019) und Informationen nach Miegel et al. (2016) in Bezug auf die Verdichtung des Torfes unterhalb der Düne sowie Dahms (1991) in Verbindung mit Messungen nach Foth (1989) in Bezug auf die Unterteilung in einen oberen, besseren durchlässigen Torf und einen unteren, schlechter durchlässigen Torf.)

Fig. 1 b Simplified plan view of the hydrogeological model zones. c Simplified geological cross-section of the coastal fen (orange line) from the Baltic Sea towards the edge of the forest including the adjacent observation points. (Modified from Jurasinski et al. (2018) and Ibenthal (2019), as well as according to Miegel et al. (2016) in relation to peat compaction below the dune and Dahms (1991) in connection with measurements according to Foth (1989) in relation to the subdivision into an upper, higher permeable peat and a lower, less permeable peat.)

2016), so auch im Januar 2019 (www.baltic-transcoast.unirostock.de). Durch Einstellung des Küstenschutzes und der infolge Klimawandel steigenden Meerespegel ist in Zukunft eine stärkere Beeinflussung durch eindringendes Ostseewasser zu erwarten. Im südlichen Teil der Niederung erhebt sich inselartig mit dem eigentlichen „Hütelmoor“ ein ca. 40 ha großes Hochmoor $(0,6 \mathrm{~m}$ ü. NHN). Im Inlandsbereich umfasst das Untersuchungsgebiet Waldflächen der Rostocker Heide ( 0,5 bis $2 \mathrm{~m}$ ü. NHN), dem höchstgelegenen Teil des NSGs (Miegel et al. 2016).

Das oberirdische Einzugsgebiet der Niederung entwässert über zahlreiche Gräben in den Prahmgraben, der an der südlichen Modellgrenze aus dem Untersuchungsgebiet (Abb. 1a) und von dort binnenseitig über den Breitling zur Ostsee ausströmt. Die in den Waldgebieten im Südosten entspringenden Gräben fallen i.d.R. im Sommer trocken. Das raue Hornblatt (Ceratophyllum demersum) führt in den langsam fließenden Niederungsgräben zu einer starken Verkrautung (Miegel et al. 2016).

Im Rahmen der Renaturierungsmaßnahmen zur Wiedervernässung des Niedermoores nach vorhergehender langjähriger Entwässerung wurde das Schöpfwerk stillgelegt und 2009 am südlich liegenden Gebietsauslass eine Sohlschwelle mit einer Höhe von ca. 0,55 m ü. NHN errichtet (Abb. 1a, zwischen HM-12 und HM-13) (Miegel et al. 2016).

In weiten Teilen der Niederung existiert eine geologische Folge von Torf über Feinsand bis hin zu Geschiebemergel, mit Mittelsand im Bereich der Düne (Abb. 1c). Abweichungen von dieser dominierenden Folge treten vor allem in lokalen Senken der Sandschichtoberkante auf, hier ist unterhalb des Moorkörpers Mudde (Gyttja) anzutreffen. Diese Ablagerungen sind im Bereich des Heiligensees stärker ausgebildet und weisen zusätzlich eine Schichtung verschiedener Muddearten auf, die sich durch eine geringe vertikale hydraulische Durchlässigkeit auszeichnen (Langguth und Voigt 1980; Krüger 1995; Ibenthal 2019).

Die Mächtigkeit des Moorkörpers schwankt zwischen 3,0 m im küstennahen Bereich bis lediglich 0,2 $\mathrm{m}$ im östlichen Bereich des Untersuchungsgebiets. Bedingt durch die frühere Entwässerung des Gebietes ist der Moorkörper besonders in den oberen Schichten stark degradiert, teilweise durchwurzelt und zusammengeschrumpft (Dahms 1991; Miegel et al. 2016; Kreuzburg et al. 2018). Der Durchlässigkeitsbeiwert in den oberen Torfschichten bis zu einer Tiefe von ca. $0,45 \mathrm{~m}$ liegt in der gleichen Größenordnung wie der des Sandaquifers und nimmt mit der Tiefe ab (Foth 1989; Dahms 1991). Es wird davon ausgegangen, dass einige Gräben stellenweise die gering leitfähigen Torfschichten durchschneiden und bis in den tiefer gelegenen Feinsandaquifer reichen (Abb. 1c). Im Bereich der höher gelegenen Waldflächen sind dagegen keine Torfablagerungen vorhanden. Der Aquifer wird im Liegenden durch den Geschiebemergel begrenzt, welcher i.d.R. gering durchlässig ist (Dahms 1991; Ibenthal 2019). 


\section{Hydrologisches Messnetz, Grundwasserneubildung}

Seit 2009 werden an 17 Beobachtungspunkten Wasserstand (h) und Temperatur (T) mit 15-minütiger Auflösung kontinuierlich erfasst, an einigen davon auch die elektrische Leitfähigkeit (EC) des Grund- und Oberflächenwassers (Abb. 1a). Ein detaillierter Überblick über die Messeinrichtungen, deren Messgrößen und zeitlichen Auflösungen ist durch Miegel et al. (2016) beschrieben.

Die Grundwassermessstellen HM-01, HM-02, HM-11 sowie P-1 bis P-5 liegen in der Niederung nicht in unmittelbarer Nähe zu einem der Gräben und sind im Torf verfiltert. Dahingegen befinden sich die Oberflächengewässerpegel HM-03 bis HM-07 und HM-10 jeweils in einem bzw. nahe eines Grabens. HM-12 ist im Prahmgraben kurz oberhalb und HM-13 direkt unterhalb der Sohlschwelle positioniert, beide Messstellen sind Oberflächengewässerpegel (Abb. 1a). Im Waldgebiet liegen die Grundwassermessstellen HM-08, HM-09, HM-14 und HM-15 mit direkter Anbindung an den Sandaquifer.

\section{Methodik}

Mittels der Kopplung eines Grundwassermodells (FEFLOW 7.1) mit einem hydrodynamischen Oberflächenwassermodell (MIKE 11) wurde der Wasserhaushalt und die Strömungssituation im Untersuchungsgebiet gesamtheitlich analysiert und visualisiert. Verschiedene Grundwassermodelle wurden schrittweise, jeweils automatisch kalibriert. Zuerst erfolgte die Kalibrierung der Durchlässigkeitsbeiwerte eines stationären und anschließend eines instationären nicht-gekoppelten, dichteunabhängigen Modells mit dem in FEFLOW integrierten Programm FePEST (DHI 2015). Hierzu wurden die Abweichungen zwischen gemessenen und simulierten Druckhöhen der Beobachtungspunkte HM-01, HM-02 und HM-11 mit der Methode der kleinsten Quadrate (MKQ) minimiert (DHI 2015). Die jeweiligen Kalibrierungsgrößen werden in der Beschreibung zum Modellaufbau näher erläutert.

Die Simulation der Oberflächenwasserströmungs- und Transportmodellierung im instationären, gekoppelten Modell erfolgte mit MIKE 11 (DHI 2017). In MIKE 11 wird die Strömung durch die eindimensionale Kontinuitätsund die Energiegleichung, den sogenannten Saint-VenantGleichungen, beschrieben. Die Kopplung von FEFLOW und MIKE 11 erfolgte durch das Schnittstellenmodul IfmMIKE 11 (Monninkhoff 2014). Der Austauschfluss zwischen FEFLOW und MIKE 11 ergab sich aufgrund der Differenz der Wasserdruckhöhe zwischen Graben und Grundwasser, der Kontaktfläche sowie einem linearen Leakage-Koeffizienten, welcher neben den Manning-
Koeffizienten im Zuge der Kalibrierung des transienten, gekoppelten Modells optimiert wurde.

Die Transportmodellierung zum Eintrag von Brackwasser erfolgte für ein transientes, kurzzeitiges Sturmhochwasser in 2017, bei dem Ostseewasser über die Sohlschwelle in die Niederung einströmte (siehe Miegel et al. 2017). Da für dieses Ereignis die hydraulische Situation stark von den sonstigen Gegebenheiten abwich, wurden für diese Modellvariante spezifische Modellanpassungen vorgenommen. Da diese jedoch schon auf den vorhergehenden Modellergebnissen aufbauen, werden diese erst in dem entsprechenden Abschnitt zum Sturmhochwasserereignis detailliert erläutert.

\section{Modellaufbau Grundwasser - FEFLOW}

Das Modellgebiet wurde in FEFLOW 7.1 aufgebaut. Es umfasst eine Fläche von ca. $5 \mathrm{~km}^{2}$. Die horizontale räumliche Diskretisierung erfolgte durch 769.245 trianguläre Elemente und die vertikale Diskretisierung durch sechs Ebenen. Die Geländeoberkante basiert auf einem DGM (GEOBASIS-DE und M-V 2018; Jonas 2019). Zusätzlich wurden Höhenangaben von Dahms (1991) berücksichtigt. Der hydrogeologische Aufbau des Modells wird durch zwischen den sechs Ebenen liegenden fünf Schichten mit elf verschiedenen hydrogeologischen Modellzonen erfasst, wobei den Modellzonen, wie z.B. Sand, jeweils homogene hydraulische Parameter zugeordnet worden sind (Tab. 1, Abb. 1b,c). Die räumliche Verteilung der hydrogeologischen Modellzonen für jede Modellschicht ist in Schreiber (2019) beschrieben. In der Niederung repräsentiert die oberste Modellschicht die dichte Schilfvegetation, in der angenommen wird, dass in dieser der Austausch von Wasser ähnlich wie in einem grobkörnigen porösen Medium erfolgt. Sie wurde als eine phreatische Aquiferschicht mit einer räumlich und zeitlich konstanten Schichtdicke von $1,5 \mathrm{~m}$ definiert. So kann ein lateraler Wasseraustausch für variable Wasserstände stattfinden, der aber den im Vergleich zu den Gräben höheren Strömungswiderstand des Schilfs berücksichtigt.

Die hydraulischen Randbedingungen der Modell-Nordwestgrenze (Ostsee) und der Inlandsseite wurden als Randbedingung erster Art entsprechend der gemessenen Wasserstände der Ostsee (Pegel Warnemünde, WSA Stralsund 2018) bzw. der dortigen Grundwassergleichen (Rechenbach 2009; LUNG M-V 2018) in täglicher Auflösung für den Kalibrierungs- und Validierungszeitraum definiert (Abb. 1a). Den Gräben in der Niederung oberhalb der Sohlschwelle wurde ein räumlich konstanter Wert, der sich am zentral gelegenen Pegel HM-10 orientiert, zugewiesen. Der Wasserstand in den Waldgräben beruht für das nicht-gekoppelte Grundwassermodell auf einer modellinternen linearen Interpolation der jeweiligen Randbedingung mit den 
Tab. 1 Hydraulische Parameter der hydrogeologischen Modellzonen im transienten Modell mit Angabe des für die Kalibrierung festgelegten Schwankungsbereiches des horizontalen Durchlässigkeitsbeiwerts (Minimum und Maximum $\mathrm{k}_{\mathrm{fh}}$ ), finaler Durchlässigkeitsbeiwert im Modell ( $\left.\mathrm{k}_{\mathrm{fh}}\right)$ und finaler Modellwert, Anisotropieverhältnis zwischen horizontalem und vertikalem Durchlässigkeitsbeiwert $\left(\mathrm{k}_{\mathrm{fh}} / \mathrm{k}_{\mathrm{fv}}\right)$, effektive Porosität $\left(n_{e}\right)$ und spezifischer Speicherkoeffizient $\left(S_{s}\right)$

Table 1 Hydraulic parameters of each hydrogeological model zone for the transient groundwater model. Parameter range set in calibration for horizontal hydraulic conductivity $\left(\mathrm{k}_{\mathrm{fh}}\right)$ and final model value, anisotropy ratio $\left(\mathrm{k}_{\mathrm{fh}} / \mathrm{k}_{\mathrm{fv}}\right)$, effective porosity $\left(n_{e}\right)$, and specific storage $\left(S_{s}\right)$

\begin{tabular}{|c|c|c|c|c|c|}
\hline Hydrogeologische Modellzonen & $\begin{array}{l}\text { Schwankungsbereich } \mathrm{k}_{\mathrm{fh}} \\
{\left[\mathrm{m} \mathrm{s}^{-1}\right]}\end{array}$ & $\begin{array}{l}\mathrm{k}_{\mathrm{fh}} \\
{\left[\mathrm{m} \mathrm{s}^{-1}\right]}\end{array}$ & $\begin{array}{l}\mathrm{k}_{\mathrm{fh}} / \mathrm{k}_{\mathrm{fv}} \\
{[-]}\end{array}$ & $\begin{array}{l}\mathrm{n}_{\mathrm{e}} \\
{[-]}\end{array}$ & $\begin{array}{l}\mathrm{S}_{\mathrm{s}} \\
{\left[\mathrm{m}^{-1}\right]}\end{array}$ \\
\hline$\overline{\text { Schilf }}$ & - & $8,1 \cdot 10^{-1}$ & 0,9 & 0,80 & $1,0 \cdot 10^{-1}$ \\
\hline oberer Torf der Niederung & $4,6 \cdot 10^{-6}-2,0 \cdot 10^{-4}$ & a $5,2 \cdot 10^{-5}$ & 2,0 & 0,30 & $9,8 \cdot 10^{-2}$ \\
\hline unterer Torf der Niederung & $4,6 \cdot 10^{-6}-2,0 \cdot 10^{-4}$ & ${ }^{a} 3,0 \cdot 10^{-5}$ & 3,0 & 0,20 & $9,8 \cdot 10^{-2}$ \\
\hline oberer Torf unterhalb der Düne & $1,0 \cdot 10^{-9}-1,0 \cdot 10^{-4}$ & ${ }^{a} 2,8 \cdot 10^{-7}$ & 2,0 & 0,30 & $9,8 \cdot 10^{-2}$ \\
\hline unterer Torf unterhalb der Düne & $1,0 \cdot 10^{-9}-1,0 \cdot 10^{-4}$ & ${ }^{\mathrm{a}} 9,2 \cdot 10^{-9}$ & 3,0 & 0,20 & $9,8 \cdot 10^{-2}$ \\
\hline oberer Torf im Hochmoor & - & $1,0 \cdot 10^{-4}$ & 2,5 & 0,30 & $9,8 \cdot 10^{-2}$ \\
\hline unterer Torf im Hochmoor & - & $2,3 \cdot 10^{-5}$ & 3,5 & 0,20 & $9,8 \cdot 10^{-2}$ \\
\hline Dünensand und mariner Sand & $2,0 \cdot 10^{-7}-5,0 \cdot 10^{-4}$ & ${ }^{a} 2,2 \cdot 10^{-4}$ & 10,0 & 0,23 & $1,5 \cdot 10^{-4}$ \\
\hline Mudde mit geringer Mächtigkeit & - & $1,2 \cdot 10^{-8}$ & 2,0 & 0,05 & $2,6 \cdot 10^{-3}$ \\
\hline Mudde mit hoher Mächtigkeit & - & $1,2 \cdot 10^{-8}$ & 3,0 & 0,05 & $2,6 \cdot 10^{-3}$ \\
\hline Sand & $3,5 \cdot 10^{-6}-5,0 \cdot 10^{-5}$ & ${ }^{a} 2,1 \cdot 10^{-5}$ & 10,0 & 0,21 & $1,5 \cdot 10^{-4}$ \\
\hline
\end{tabular}

${ }^{\mathrm{a}}$ Kalibrierte Werte/calibrated values

Wasserständen in den Niederungsgräben. Fehlende Wasserstandsdaten einzelner Messstellen wurden aus Werten des gleichen Pegels anderer Jahre hergeleitet bzw. linear zwischen den verfügbaren Werten derselben Zeitreihe interpoliert. Die beiden orthogonal zur Ostsee verlaufenden Modellgrenzen im Nordosten und Südwesten entsprechen näherungsweise den lokalen Grundwasserströmungslinien. Sie wurden daher, wie auch der Geschiebemergel als untere Modellgrenze, als undurchlässiger Rand definiert. Am oberen Rand wird als Randbedingung flächig die klimatische Wasserbilanz angesetzt. Diese Randbedingung stellt somit eine Quelle bzw. Senke an der Oberkante vom Aquifer dar und entspricht je nach saisonalen Gegebenheiten einer Grundwasserspeisung bzw. -zehrung.

Die klimatische Wasserbilanz, welche in nicht überstauten Arealen der Grundwasserneubildung entspricht, wurde basierend auf den Daten der DWD-Klimastation Rostock-Warnemünde (DWD 2018a, b) als Differenz zwischen Niederschlag und realer Evapotranspiration berechnet (Hölting und Coldewey 2013). Die Berechnung der Evaporation von Wasserflächen erfolgt mit dem Dalton-Verfahren (DVWK 1996) mit Werten der Windfunktionskoeffizienten nach Richter (1977). Der Rauhigkeitskoeffizient beträgt für das Gebiet 0,15 (Miegel et al. 2016).

Die potenzielle Evapotranspiration $\left(\mathrm{ET}_{\mathrm{pot}}\right)$ wurde auf Grundlage der potenziellen Grasreferenzverdunstung nach Penman-Monteith (DWD 2018a) berechnet, und zwar separat für die Teilflächen Wald (Bestandskoeffizient=1,2), Schilf, Düne und Strand. Aufgrund der guten Wassernachlieferung in der Niederung entspricht bei Schilf, Strand und Düne die reale Evapotranspiration der potenziellen (Miegel et al. 2016). Für die Wald-Standorte wird von einem Faktor von 0,8 zwischen potenzieller und realer Evapotranspiration ausgegangen (Miegel et al. 2016).

Bei der Transportmodellierung für eindringendes salziges Ostseewasser wurden in FEFLOW direkt EC-Werte verwendet und dementsprechende Randbedingungen festgelegt. So betragen diese an der Modell-Nordwestgrenze (Ostsee) und Inlandsseite gemäß mehrerer Stichtagsmessungen $19,6 \mathrm{mS} \cdot \mathrm{cm}^{-1} \mathrm{bzw}$. für die Inlandsseite $0,2 \mathrm{mS} \cdot \mathrm{cm}^{-1}$ (Krüger 1995). Die Anfangskonzentration in der Niederung wurde aufgrund entsprechender manueller Feldmessungen mit $9,6 \mathrm{mS} \cdot \mathrm{cm}^{-1}$ definiert. Die im Ostseewasser dominierenden Ionen sind Natrium und Chlorid (Kulik und Harff 1993). Natrium unterliegt Ionenaustauschprozessen, wohingegen Chlorid meist als konservativer Tracer angesehen wird (Brucher 2007; Hölting und Coldewey 2013). Als molekularer Diffusionskoeffizient wurde als vereinfachte Näherung der Wert von Chlorid in Wasser mit $1,8 \cdot 10^{-9} \mathrm{~m}^{2} \cdot \mathrm{s}^{-1}$ gewählt (Poisson und Papaud 1983). Die longitudinale Dispersivität wurde auf ein Zehntel der Dimension der Fließstrecke festgelegt. Es wurde angenommen, dass das Salzwasser ca. $10 \mathrm{~m}$ von den Gräben in die Fläche strömt, da die Messstelle P-3 etwa $15 \mathrm{~m}$ von einem Graben entfernt liegt und hier keine Erhöhung der elektrischen Leitfähigkeit gemessen wurde. Dementsprechend wurde der longitudinalen Dispersivität ein Wert von $1 \mathrm{~m}$ und der transversalen Dispersivität mit $0,1 \mathrm{~m}$ ein Zehntel dieses Wertes zugeordnet (Gelhar et al. 1992).

\section{Modellaufbau Grabensystem - MIKE 11 und Modellkopplung}

Das Grabensystem wurde in ArcGIS anhand von digitalen Orthofotos (LAiV M-V \& AfGVK M-V 2018) so- 
wie einem DGM mit einer horizontalen Auflösung von $1 \mathrm{~m}$ (GEOBASIS-DE und M-V 2018; Jonas 2019) digitalisiert. Im Untersuchungsgebiet befinden sich insgesamt 52 Gräben mit einer Länge zwischen $52 \mathrm{~m}$ und $3036 \mathrm{~m}$. Die mittlere Sohlhöhe der Gräben in der Niederung beträgt $-1,1 \mathrm{~m}$ ü. NHN, lediglich der Prahmgraben hat eine niedrigere mittlere Sohlhöhe von -1,9m ü. NHN (Dahms 1991). Die Grabenquerschnitte wurden aus dem DGM abgeleitet und anhand manueller Messungen von Dahms (1991) korrigiert. Die Sohlschwelle ist als Wehr mit einer Höhe von $0,55 \mathrm{~m}$ ü. NHN implementiert. Bei den Manning-Koeffizienten wird zwischen den Gräben in der Niederung und den Gräben im Wald unterschieden. Sowohl die Manning-Koeffizienten als auch der Leakage-Koeffizient wurden mittels der MKQ an den Beobachtungspunkten HM-01, HM-02, HM-06 und HM-11 manuell kalibriert.

Als transiente Randbedingung wurde den Grabenenden (Punkte der Gräben, welche in eine Richtung keine Verbindung aufweisen) der Niederung und der landeinwärts befindlichen Waldgräben der 12.00 Uhr-Wasserstand der Messstellen HM-10/HM-13 (für oberhalb/für unterhalb der Sohlschwelle) bzw. HM-09 zugeordnet. Basierend auf den Randbedingungen werden die Anfangsbedingungen von MIKE 11 linear interpoliert (Monninkhoff 2014).

Für das Sturmhochwasserereignis (vom 01. bis 11.01. 2017) wurden die entsprechenden Randbedingungen alle Stunde bzw. $15 \mathrm{~min}$ (vom 04. bis 05.01.2017) vorgegeben, um den transienten Verlauf der Flutwelle akkurat abbilden zu können. Um die homogenen Wasserstände der Niederung zu realisieren wurde dem Ende des Waldrandgrabens zusätzlich der Wert von Rohr HM-10 zugeordnet.

Die gemessenen Grabenwasserstände während des Sturmhochwassers wurden in der Simulation, vermutlich aufgrund des zu geringen oberflächigen Abstroms in die Niederung, deutlich überschätzt. Um den Wasseraustausch zwischen den Gräben und der Niederungsfläche zu verbessern, wurde für die Gräben in FEFLOW oberhalb des Torfes eine zusätzliche Modellschicht mit hohen Durchlässigkeitsbeiwerten entsprechend der Schilfschicht eingefügt und somit der flächige Abstrom in die Niederung begünstigt. Des Weiteren wurde für die Simulation des Hochwasserereignisses der Querschnitt der Sohlschwelle und der Waldgräben verringert, um der zuvor simulierten deutlichen Überschätzung des Abflusses entgegenzuwirken.

Die elektrische Leitfähigkeit am Gebietsauslass unterhalb der Sohlschwelle (während des Sturmhochwassers strömt an dieser Stelle Brackwasser in die Niederung) wurde entsprechend des dortigen Messpunktes HM-12 definiert. Den Grabenenden im Wald und dem Ende des Waldrandgrabens wurde ein konstanter Wert von $0,2 \mathrm{mS} \cdot \mathrm{cm}^{-1}$ bzw. $0,8 \mathrm{mS} \cdot \mathrm{cm}^{-1}$ zugewiesen. Die Anfangsbedingungen der EC in den Gräben der Niederung und im Wald betragen $2 \mathrm{mS} \cdot \mathrm{cm}^{-1}$ und $0,2 \mathrm{mS} \cdot \mathrm{cm}^{-1}$ (Krüger 1995). Für die Trans- portmodellierung wurden weiterhin der Dispersionsfaktor sowie -exponent festgelegt und angepasst. Diese Parameter dienen in Verbindung mit der Strömungsgeschwindigkeit der Beschreibung der Dispersion (DHI 2017).

Die Simulation und die Ergebnisdarstellung der transienten, gekoppelten Simulation erfolgt separat für den Kalibrierungszeitraum (01.06.2010 bis 30.05.2011), den Validierungszeitraum (01.01. bis 31.12.2016) und das Sturmhochwasserereignis (01. bis 11.01.2017). Dem Kalibrierungs- und Validierungszeitraum geht jeweils eine Vorlaufphase (Warmup Periode) von zwei Monaten voraus. Als Anfangsbedingungen für den Kalibrierungs- und Validierungszeitraum dienen die Simulationsergebnisse des stationären Modells.

\section{Ergebnisse und Diskussion}

\section{Modellkalibrierung und Validierung}

Die kalibrierten Parameter für die hydrogeologischen Modellzonen sind in Tab. 1 mit Angabe des Schwankungsbereiches aufgeführt. Der Fehler in der Wasserbilanz betrug lediglich 0,01\%. Die Durchlässigkeitsbeiwerte der Muddeschichten im Hochmoor wurden nicht kalibriert, da diese lediglich einen sehr lokalen Einfluss auf das Strömungsverhalten zeigten. Die geringen Durchlässigkeitsbeiwerte des Torfes unterhalb der Düne korrespondieren mit der Annahme, dass dieser während des Dünenbaus stark verdichtet und zusammengepresst wurde (Miegel et al. 2016). Die kalibrierten Manning-Koeffizienten für die Gräben im Wald/ Niederung und der lineare Leakage-Koeffizient in der Niederung betragen $0,10 \mathrm{~s} \cdot \mathrm{m}^{-\frac{1}{3}} / 0,12 \mathrm{~s} \cdot \mathrm{m}^{-\frac{1}{3}}$ bzw. $200 \mathrm{~d}^{-1}$. Die kalibrierte Austauschrate für das FEFLOW-MIKE 11-Modell ist mit $200 \mathrm{~d}^{-1}$ relativ hoch, was jedoch durch die hohe Leitfähigkeit der angrenzenden Schilfschicht bedingt wird. Durch diese breitet sich ein Großteil des Wassers in der Niederung aus.

Die Summe der Fehlerquadrate (MKQ) zeigt unter Verwendung von HM-02, HM-06 und HM-11 für den Kalibrierungszeitraum $\left(\mathrm{MKQ}=0,86 \mathrm{~m}^{2}\right)$ eine bessere Übereinstimmung als für den Validierungszeitraum $\left(\mathrm{MKQ}=3,96 \mathrm{~m}^{2}\right)$ (von HM-01 lagen im Validierungszeitraum keine Messwerte mehr vor). Dies bestätigt sich auch unter Betrachtung der gemittelten Fehlerquadrate (RMSE) (Kalibrierung: 0,01-0,04 m, Validierung: 0,05-0,07 m). Das Bestimmtheitsmaß $\left(\mathrm{R}^{2}\right)$ hingegen zeigt für die Messstellen HM-02 und HM-11 eine bessere Korrelation von jeweils 0,96 im Validierungszeitraum im Vergleich zu 0,94 und 0,92 im Kalibrierungszeitraum. Insgesamt stimmen die transienten Simulationsergebnisse des gekoppelten Grundwasser-Oberflächenwassermodells sehr gut mit den gemessenen Werten überein (Abb. 2) und bilden die saisonalen Schwankungen 


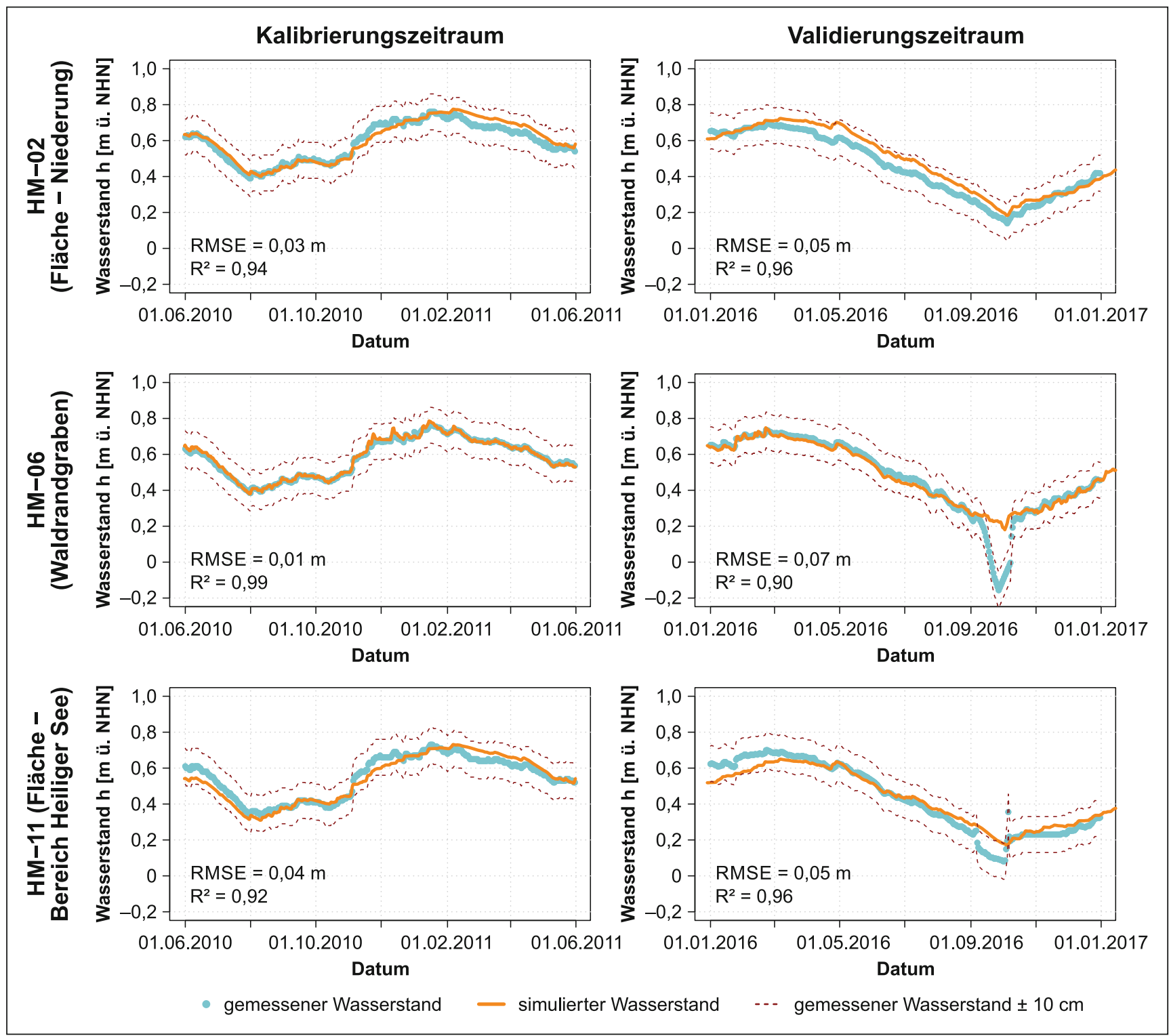

Abb. 2 Gemessene (hellblaue Punkte) und simulierte Wasserstände der Niederung (HM-02, HM-11) und der Gräben (HM-06) für den Kalibrierungs- (links) und Validierungszeitraum (rechts) für das gekoppelte Grundwasser-Oberflächenwassermodell (orange Linie) inklusive der mittleren quadratischen Abweichung (RMSE) und des Bestimmtheitsmaßes $\left(\mathrm{R}^{2}\right)$

Fig. 2 Measured (turquoise points) and simulated (orange line) water levels in groundwater (HM-02, HM-11) and surface water (HM-06) for the calibration (left) and validation period (right) of the coupled FEFLOW-MIKE 11 model. The root mean square error (RMSE) between measured and simulated values as well as determination coefficient $\left(\mathrm{R}^{2}\right)$ are displayed in the lower-left corner of each subfigure

der Wasserstände weitestgehend ab. Die starke Absenkung des Grabenwasserstandes bei den Messstellen HM-06 und HM-11 im September/Oktober 2016 kann aufgrund der gewählten Randbedingungen nicht abgebildet werden. Durch die starke Absenkung des Wasserstands im Waldrandgraben wurde vermutlich sein Austausch mit dem sonstigen Grabensystem unterbrochen, so wie das auch am Heiligensee beobachtet werden kann. Die starke Absenkung wird unter dieser Bedingung möglicherweise durch die Zehrung der benachbarten Bäume und einen sich temporär umkehrenden Gradienten in Richtung Wald begünstigt, also Phänomene, die durch das aktuelle Modell nicht abgebildet werden können und erst noch näher untersucht werden müssten.

\section{Hydraulische Situation in der Niederung}

Abb. 3 zeigt die Druckverteilungen zweier repräsentativer Zeitpunkte in der ersten Modellebene, zu denen der Wasserstand im Graben über $(0,67 \mathrm{~m}$ ü. NHN am 01.04.2016) und unter $(0,46 \mathrm{~m}$ ü. NHN am 31.12.2016) dem Höhenniveau der Sohlschwelle $(0,55 \mathrm{~m}$ ü. NHN) lag. Weitere Druckverteilungen des Kalibrierungszeitraumes (alle zwei Monate) 


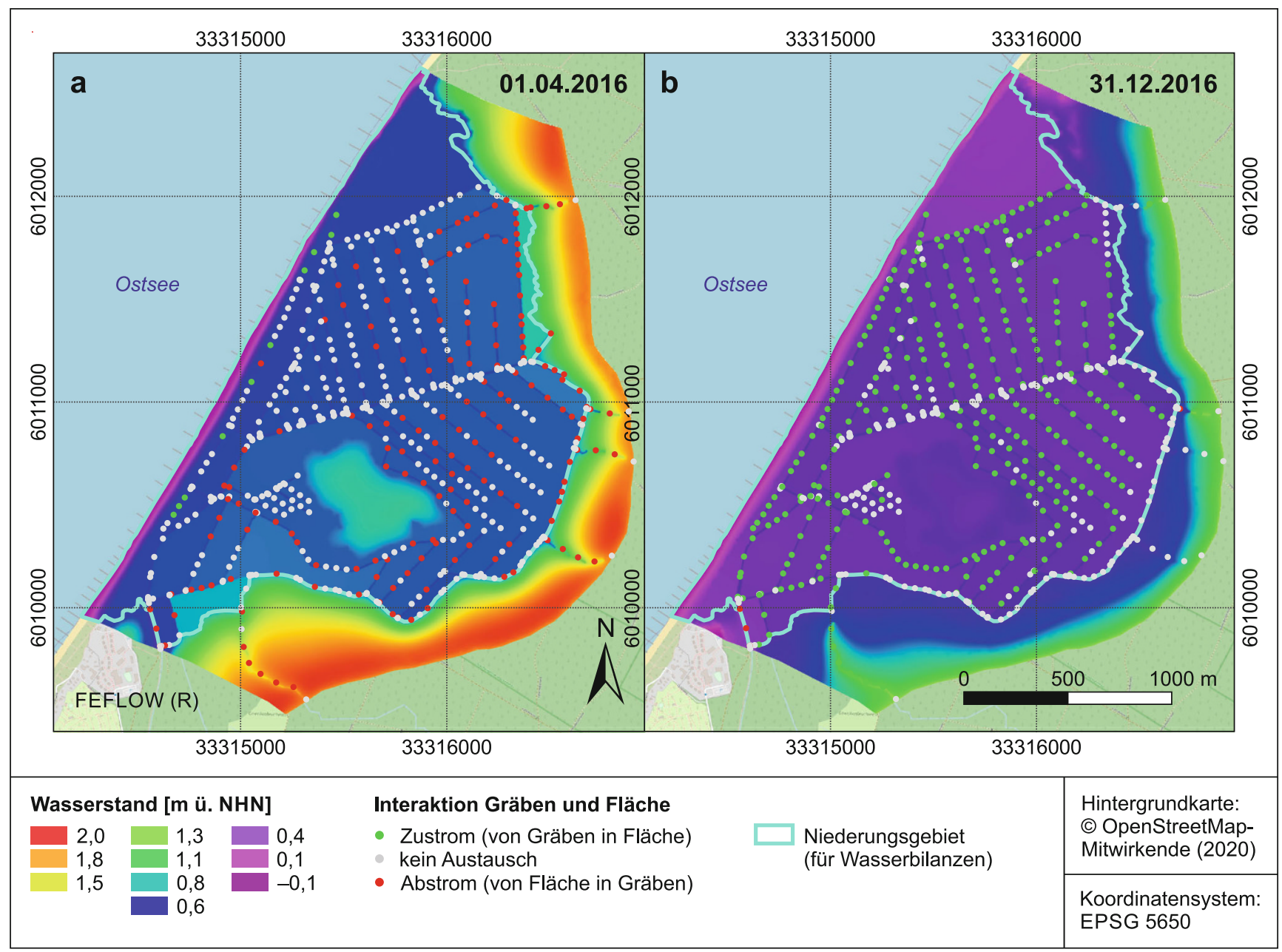

Abb. 3 Druckverteilung für repräsentative Verhältnisse a der Abfluss- sowie b der Kein-Abfluss-Phase des Oberflächenwassers über die Sohlschwelle

Fig. 3 Representative distribution of pressure head of the uppermost FEFLOW layer for „discharge condition“ (a) and „,no discharge condition“ (b) of the surface water over the bed sill at the catchment outlet

sind in Schreiber (2019) dargestellt. Zu ca. 58\% der Tage eines Jahres (im Mittel über die Jahre 2012 bis 2017) lag der Wasserstand im Graben unterhalb (Kein-AbflussPhase) und zu $42 \%$ oberhalb der Sohlschwelle. Der zweite Fall bedeutet, dass Grabenwasser über die Sohlschwelle aus dem Untersuchungsgebiet abfließt (Abfluss-Phase), ausgenommen im Falle von Sturmhochwassern.

Diese beiden Phasen spiegeln deutlich die saisonalen hydrologischen Unterschiede wider, die hauptsächlich durch das Wechselspiel von Niederschlag und Verdunstung und damit die saisonal unterschiedliche Abflussbildung bedingt sind. In beiden Phasen zeigen die Grundwasserstände einen negativen Gradienten vom östlichen Rand zur Niederung (am 01.04.2016 max. 6,2 $10^{-3}$, am 31.12.2016 max. $\left.5,2 \cdot 10^{-3}\right)$. Entsprechend strömt Grundwasser aus den Waldgebieten in das Niedermoor, wobei der Waldrandgraben einen Teil des zuströmenden Wassers direkt aufnimmt. Der Druckhöhengradient und der daraus resul- tierende Grundwasserstrom innerhalb der Niederung sind sehr klein. Lokal treten leicht abweichende Wasserstände im Bereich des Hochmoores auf, welches nicht an das Grabensystem angeschlossen ist. In der Umgebung der Düne strömt das Wasser in Richtung Ostsee ab, auch wenn damit ein geringer Beitrag zum Gesamtabfluss des Gebietes verbunden ist.

Von Januar bis Anfang Juni 2016 (Abb. 3a, AbflussPhase) sind die absoluten Wasserstände deutlich höher (Inlandseite mit $1,72 \mathrm{~m}$ ü. NHN am 01.04.2016 gegenüber $1,34 \mathrm{~m}$ ü. NHN am 31.12.2016), und der Wasserstand in der Niederung und deren Gräben liegt oberhalb des Höhenniveaus der Sohlschwelle. In den Gräben strömt das Wasser Richtung Gebietsauslass, wo es das Gebiet über die Sohlschwelle verlässt. Die Wasserstände des Grundwassers bzw. im Schilf sind in dieser Phase des Validierungszeitraumes im Mittel höher als die Grabenwasserstände, wo- 
Abb. 4 Wasserbilanz für das gekoppelte Grundwasser-Oberflächenwasser-Modell im Validierungszeitraum a für Austausch Niederung mit der Ostsee sowie b für Austausch der Niederung mit den Gräben und Abfluss über die Sohlschwelle

Fig. 4 Transient exchange flux between fen and Baltic Sea (a) and surface water and groundwater (b) for the coupled groundwater-surface water model of the validation period. Green (IN) and red (OUT-flow) bars indicate the direction of the exchange flux in respect to the model domain. Discharge over the bed sill at the catchment outlet is displayed by the purple line (b)

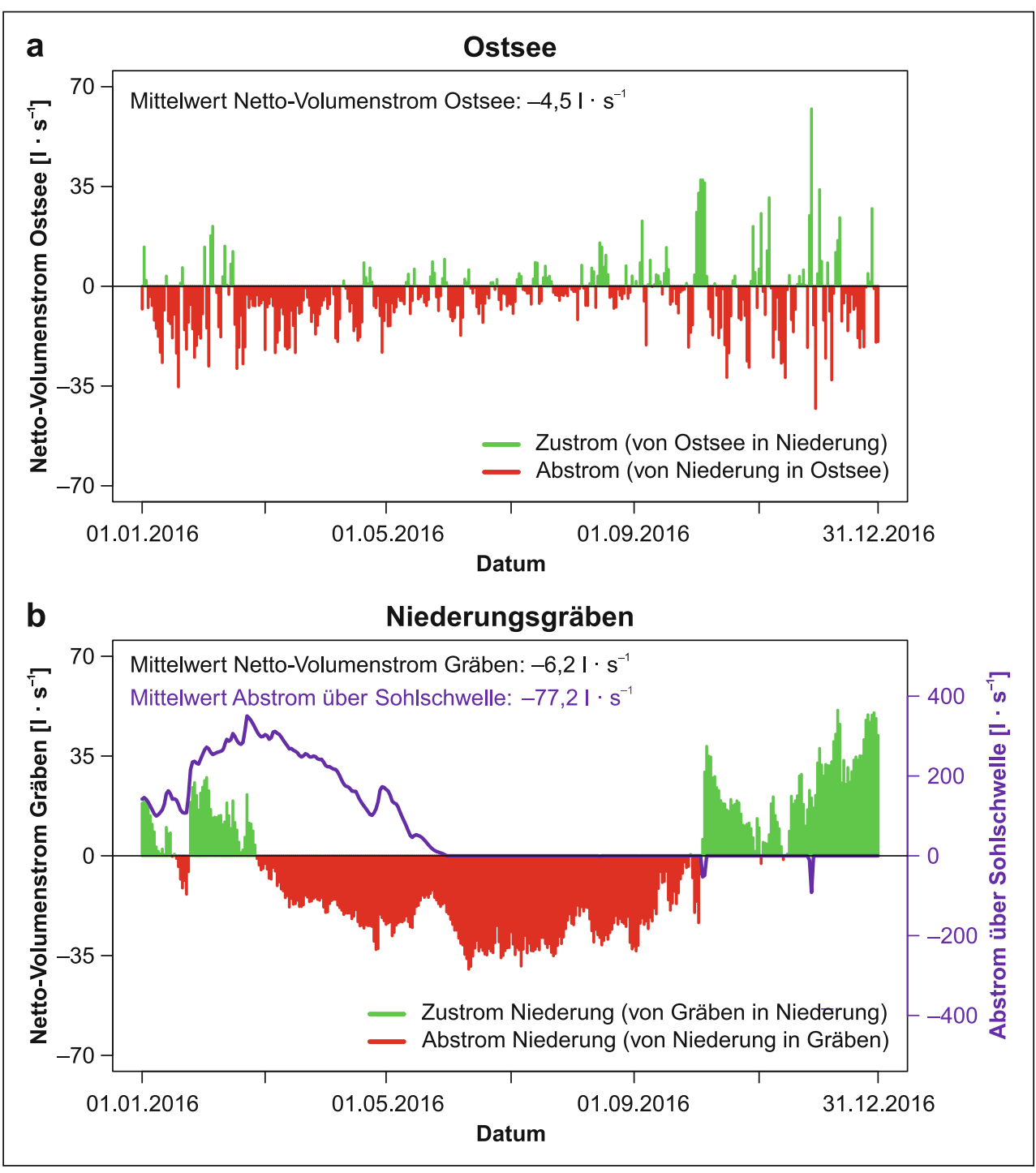

durch i.d.R. den Gräben Wasser aus der Niederung zuströmt (Abb. 3a).

Im Sommer (Abb. 3b, Kein-Abfluss-Phase) sinkt aufgrund der Dominanz der Verdunstung in der Vegetationsperiode der Wasserstand in den Gräben der Niederung unterhalb des Höhenniveaus der Sohlschwelle ab, auch weil der oberirdische Zufluss aus dem Wald ausbleibt. Bis Anfang Oktober strömt Wasser von den Niederungsflächen in die Gräben, danach kommt es zu einem Zustrom von Grabenwasser in die Niederung, u. a., weil ein oberirdischer Zufluss aus dem Wald die Gräben mit Wasser erfüllt.

Speziell der Austausch mit der Ostsee ist im Jahresverlauf, und auch innerhalb der einzelnen Phasen, stark alternierend (Abb. 4a). Beispielsweise ist am 28.11.2016 der zutretende Volumenstrom von der Ostsee (Ostseewasserspiegel $0,72 \mathrm{~m}$ ü. NHN) in den Aquifer im Validierungszeitraum am größten. Es zeigt sich bei hohen Wasserständen der Ostsee eine landwärts gerichtete Grundwasserströmung, wel- che jedoch nur bis in den Bereich der Düne reicht. Der umgekehrte Fall eines Grundwasser-Abstroms zur Ostsee tritt dagegen, zumindest nach Einbau der Sohlschwelle, häufiger auf. Das maximal ausströmende Volumen im Validierungszeitraum ergab sich in der Simulation für den 30.11.2016 (Ostseewasserspiegel $-0,16 \mathrm{~m}$ ü. NHN). Das Wasser strömt dabei v.a. durch den unteren Sand sowie den Dünensand und marinen Sand in die Ostsee. Zwischen dieser hydraulischen Veränderung von maximalem Grundwasserzustrom $\left(+62 \mathrm{l} \cdot \mathrm{s}^{-1}\right) \mathrm{zu}$ maximalem Abstrom $\left(-431 \cdot \mathrm{s}^{-1}\right)$ lagen lediglich 2 Tage. Dies zeigt das dynamische Verhalten entlang der Küste, welches, neben den saisonalen Variationen bedingt durch die klimatische Wasserbilanz, deutlich durch die kurzzeitigen Wasserspiegelschwankungen der Ostsee bestimmt wird. Auch der Pearson-Korrelationskoeffizient zwischen Ostseewasserstand und Grundwasserzustrom in die Ostsee zeigt mit einem Wert von 0,67 (Kalibrierungs- 
zeitraum) und 0,81 (Validierungszeitraum) einen deutlich positiven Zusammenhang auf.

Der akkumulierte Nettoaustausch zwischen den Niederungsgräben und der Schilfschicht sowie dem angrenzenden Grundwasser (Abb. 4b) besitzt eine geringere zeitliche Dynamik als der Austausch zwischen Ostsee und Grundwasser, weist jedoch eine größere räumliche Variabilität auf. Besonders im Bereich der Düne infiltriert Wasser von den Gräben in die Niederung, um von hier weiter in Richtung Ostsee zu strömen. Der Waldrandgraben nimmt hingegen i.d.R. Wasser, auch aus dem Waldgebiet, auf oder zeigt keine Interaktion. Innerhalb der Niederung wechseln sich infiltrierende bzw. exfiltrierende Bedingungen, welche in Abhängigkeit von der Entwicklung der Grabenwasserstände stehen, mit Bedingungen ab, unter welchen kein Austausch stattfindet (Abb. 3a,b).

\section{Wasserbilanz der Niederung}

Die Wasserbilanz der Niederung setzt sich aus dem oberund unterirdischen Zufluss aus dem Wald (Q-OFW Waldgräben

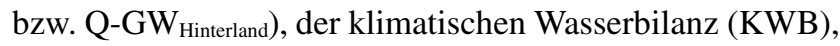
dem Abstrom über die Sohlschwelle (Q-OFW Sohlschwelle $_{\text {) }}$ und dem Grundwasseraustausch mit der Ostsee (Q-GW $\mathrm{GWer}_{\text {Mu- }}$ zusammen (Tab. 2).

Die klimatische Wasserbilanz für den Kalibrierungs- und Validierungszeitraum beträgt $6,94 \mathrm{l} \cdot \mathrm{s}^{-1}(2010 / 2011)$ bzw. $-12,53 \mathrm{l} \cdot \mathrm{s}^{-1}$ (2016). Laut den Ergebnissen der Modellierung strömt durch den oberirdischen Zufluss aus dem Wald ein großer Teil des Wassers in die Niederung und von dort aus über das Grabensystem direkt zum Gebietsauslass. Der Zufluss wurde als Restglied der Wasserbilanz bestimmt, da die Randbedingungen des Modells innerhalb der Niederung das so zuströmende Wasser zum Teil selbst aufnehmen. Das oberirdisch zuströmende Wasser beeinflusst im

Tab. 2 Wasserbilanz der Niederung für den Kalibrierungs- und Validierungszeitraum (ohne Speicheränderung). Positive Werte entsprechen einem netto Zustrom in die Niederung, negative einem Abstrom

Table 2 Water balance of the coastal fen considering climatic water balance (KWB), groundwater flow (Q-GW) and surface water flow (Q-OFW) for the calibration (left) and validation period (right). Positive values correspond to a net inflow into the valley, negative values to an outflow

\begin{tabular}{|c|c|c|}
\hline & $\begin{array}{l}\text { Juni 2010-Mai } \\
2011\end{array}$ & $\begin{array}{l}\text { Jan. 2016-Dez } \\
2016\end{array}$ \\
\hline $\mathrm{KWB}\left[1 \cdot \mathrm{s}^{-1}\right]$ & 6,9 & $-12,5$ \\
\hline Niederschlag $\left[1 \cdot \mathrm{s}^{-1}\right]$ & 78,6 & 59,8 \\
\hline Evapotranspiration $\left[1 \cdot \mathrm{s}^{-1}\right]$ & $-71,6$ & $-72,3$ \\
\hline Q-GW Hinterland $\left[1 \cdot \mathrm{s}^{-1}\right]$ & 2,2 & 1,0 \\
\hline Q-GW Meer $\left[1 \cdot \mathrm{s}^{-1}\right]$ & $-6,3$ & $-4,5$ \\
\hline Q-OFW Sohlschwelle $\left[1 \cdot \mathrm{s}^{-1}\right]$ & $-124,1$ & $-77,2$ \\
\hline Q-OFW Waldgräben $\left[1 \cdot \mathrm{s}^{-1}\right]$ & 115,4 & 72,9 \\
\hline
\end{tabular}

Modell in erster Linie den Abfluss über die Sohlschwelle und hat auf die weiteren Komponenten keinen bedeutenden Einfluss. Der unterirdische Zustrom aus dem Wald ist mit lediglich 2,21 $1 \cdot \mathrm{s}^{-1}$ bzw. 1,02 $1 \cdot \mathrm{s}^{-1}$ nur ein Bruchteil des oberirdischen Volumenstromes. Somit beträgt der $\mathrm{Zu}-$ strom von Grundwasser in die Niederung lediglich 1,3\% (2016) bis 1,7\% (2010/2011) des Gebietsabflusses. Hierdurch kann die These von Miegel et al. (2016) bekräftigt werden, dass der Gebietsabfluss zwar durch den Zustrom von Grundwasser beeinflusst, aber nicht dominiert wird.

Der Abfluss aus der Niederung erfolgt primär über die Sohlschwelle. Dieser wird hauptsächlich durch den oberirdischen Zufluss aus dem Wald aber auch durch einen Grundwasserzustrom in die Gräben gebildet. Die Simulationsergebnisse zeigen einen substanziellen Abstrom von Grundwasser zur Ostsee (Tab. 2). Der Grundwasser-Abstrom zur Ostsee beträgt 4,8\% (2010/2011) bis 5,5\% (2016) des oberirdischen Gebietsabflusses. Somit muss, im Gegensatz zu den Thesen von Miegel et al. (2016) und Selle et al. (2016), dieser Komponente eine entsprechende Bedeutung in der jährlichen Wasserbilanz des Niedermoores zugesprochen werden. Der unterirdische Volumenstrom zur Ostsee ist um das drei- bis vierfache größer als der Grundwasserzustrom in das Untersuchungsgebiet an den südöstlich gelegenen Waldgebieten. Dabei weisen die niedrigen, kalibrierten Durchlässigkeitsbeiwerte auf eine starke Verdichtung des Torfes unter der Düne hin, was plausibel ist. Somit erfolgt der Abstrom von Grundwasser zur Ostsee v. a. lokal durch den gut leitfähigen Dünensand und marinen Sand.

\section{Das Sturmhochwasserereignis und seine Eindringdynamik}

Bei dem untersuchten Sturmhochwasser strömte Brackwasser über Breitling und äußeren Prahmgraben, binnenseitig zur Sohlschwelle, über die das Gebiet normalerweise entwässert, überspülte diese massiv und strömte in die Gräben sowie von dort oberflächlich ins Gebiet ein. Der simulierte Wasserstand erreichte am 04.01.2017 um 15.30 Uhr das Höhenniveau der Sohlschwelle und sank erst am 06.01.2017 um 05.45 Uhr wieder unter dieses Niveau ab (Abb. 5, HM12). Am 08.01.2017 kam es durch einen kurzzeitigen Wiederanstieg des Wassers im Prahmgraben erneut zu einem - im Vergleich zur eigentlichen Hochwasserwelle allerdings schwachen - Zustrom in die Niederung. Bereits in den vorangegangenen Monaten war Ostseewasser bis zur Sohlschwelle vorgedrungen und hatte zu einer substanziellen Erhöhung der EC-Werte direkt an der Sohlschwelle (Abb. 5, HM-12), aber (noch) nicht in der Niederung geführt.

Die Modellergebnisse zeigen eine gute Übereinstimmung zwischen simulierten und gemessenen Druckhöhen in den Gräben und in der Niederung (Abb. 5). Die Druck- 

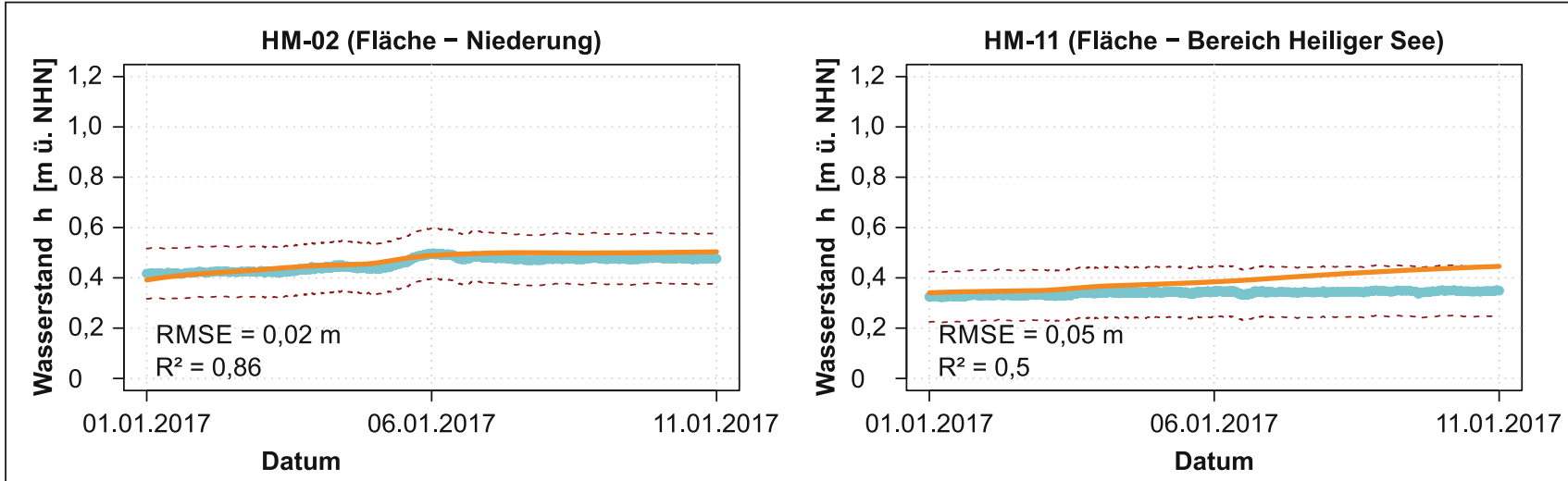

HM-12 (direkt oberh. Sohlschwelle)
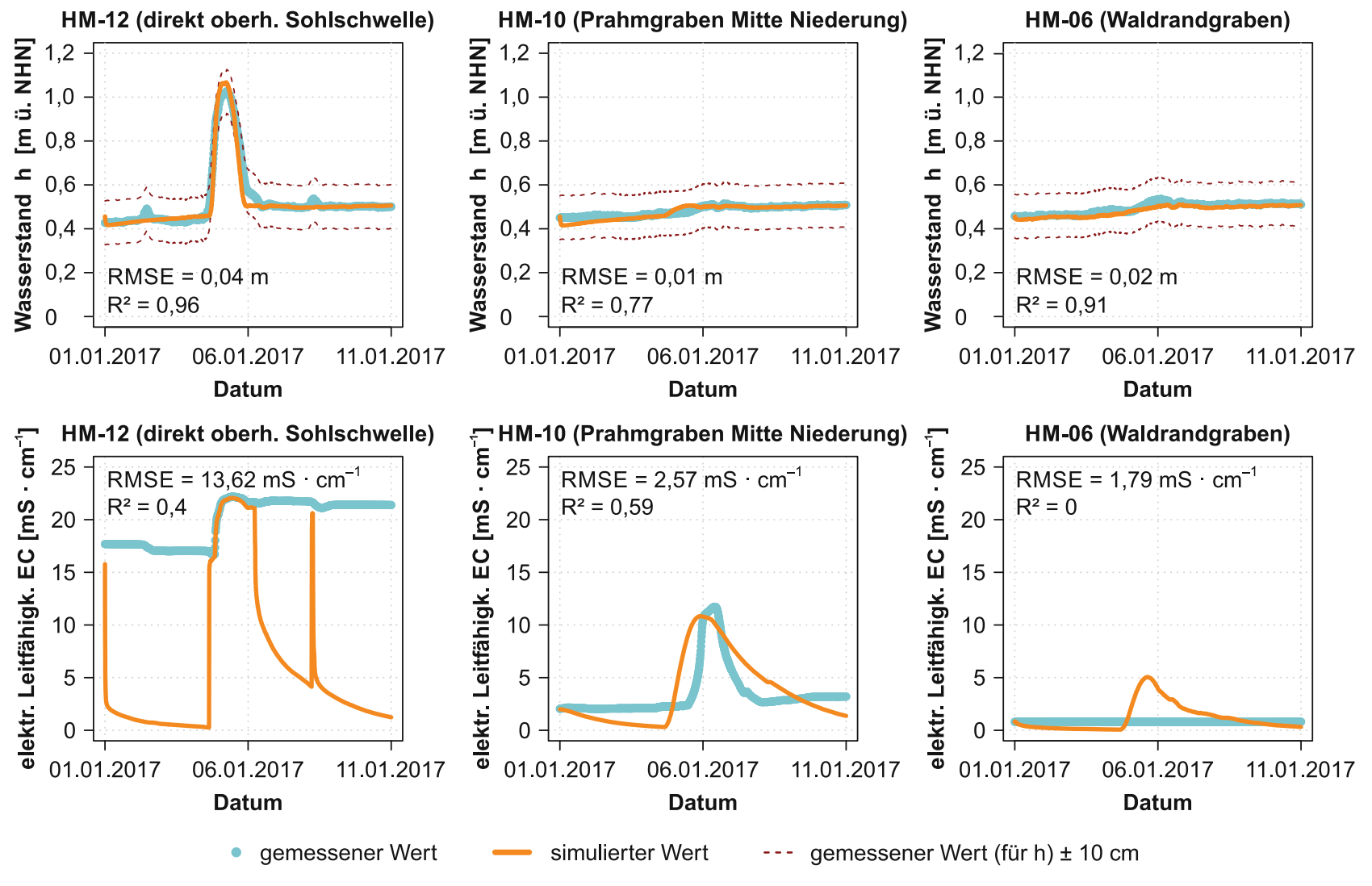

Abb. 5 Gemessene und simulierte Druckganglinien in der Niederung (oben) und den Gräben (Mitte) sowie gemessene und simulierte Konzentrationsganglinien der Gräben (unten) für das Hochwasserereignis vom 4. bis 6. Januar 2017 inklusive der mittleren quadratischen Abweichung (RMSE) und des Bestimmtheitsmaßes $\left(\mathrm{R}^{2}\right)$

Fig. 5 Measured (turquoise points) and simulated (orange line) groundwater hydraulic heads in the lowland area (top) as well as measured and simulated hydraulic heads (middle) and electrical conductivities (bottom) in the surface water for the flood event on January 4th-6th, 2017. The root mean square error (RMSE) and determination coefficient $\left(\mathrm{R}^{2}\right)$ are specified on the left of each individual diagram

höhen im Bereich des Heiligensees werden jedoch eher überschätzt (Abb. 5, HM-11). In diesem Bereich gleicht sich der Wasserstand dem mittleren Wasserstand der Niederung an. Durch das Implementieren der Schilfschicht wird der Wasserspiegel des Heiligensees künstlich angehoben, was in dieser Situation unrealistisch ist und einen lateralen Volumenstrom aus der Niederung vortäuscht, der in dem Maße real nicht stattfindet.
Während des Sturmhochwassers wurde ein Anstieg der EC vor allem im Bereich der Sohlschwelle (Abb. 5, HM12) mit bereits vorab erhöhten EC-Werten, sowie in der Niederung (Abb. 5, HM-10) gemessen. Die mithilfe dieser Messpunkte optimierten Parameter Dispersionsfaktor und Dispersionsexponent (DHI 2017) betragen 100 bzw. 0,1. Während des Eindringens der Hochwasserwelle in die Niederung (04.-06.01.2017) stimmen die simulierten und ge- 
messen EC an der Sohlschwelle gut überein (Abb. 5, HM12). Die deutlichen Abweichungen vor und nach dem eigentlichen Sturmhochwasser, an denen die gemessenen EC nahe dem Maximum verharren, während die simulierten EC wieder deutlich abfallen, liegen sehr wahrscheinlich in dem nicht berücksichtigten Dichteeinfluss des Brackwassers und der tiefen Position des EC-Sensors unterhalb der Grabensohle begründet. So kann der Stauraum vor der Sohlschwelle als Totraum betrachtet werden, in dem das salzigere Wasser aufgrund seiner Dichte absinkt, und nicht spontan abfließen kann, wenn sich die Fließrichtung wieder umkehrt, und somit lokal über längere Zeiträume zurückgehalten wird. Aus diesem Grund scheinen die EC an dem Messpunkt HM-12 im Vergleich zu den simulierten Werten durch vorangegangene als auch nach dem aktuellen Ostseehochwasser erhöht. Die Simulationen an dem Pegelrohr HM-10 in der Niederung stimmen in Hinblick auf den Zeitpunkt und den maximalen Wert sehr gut mit den gemessenen Werten überein. Abweichungen zwischen gemessenen und simulierten EC ergeben sich hauptsächlich durch ein um $19 \mathrm{~h}$ zu frühes Einsetzen des Konzentrationsanstiegs und eines deutlich langsameren Konzentrationsabfalls (längeres Tailing). Nach ca. 5 Tagen wird wieder ein niedriges Niveau der EC erreicht, das in etwa dem Ausgangswert vor Ereignisbeginn entspricht.

Für die weiteren Messstellen in der Niederung (HM02, HM-11, P-2, P-3, P-4) wurden, in Übereinstimmung mit den Messwerten, keine Erhöhungen der EC simuliert.
Eine Ausnahme bildet die Messstelle HM-06, an der sich im Gegensatz zu den Messwerten in den Simulationen eine Erhöhung der EC zeigte. Dies ist wahrscheinlich auf eine Überschätzung der Strömung in Richtung Waldgraben bzw. einer Unterschätzung der flächenhaften Salzwasserintrusion in die Gräben der Niederung zurückzuführen. Aufgrund der Schwere des Brackwassers dürfte dies hauptsächlich über den tieferen Prahmgraben in die Niederung eingeströmt sein, wie auch die Analysen von Miegel et al. (2017) vermuten lassen.

Ganz anders stellt sich die Messstelle HM-11 dar, in welcher leicht erhöhte EC gemessen, aber nicht simuliert wurden. Der Grund für den Anstieg der Salzkonzentration ist laut Miegel et al. (2017) nicht das über die Sohlschwelle einströmende Wasser, sondern ein direktes, lokales Eindringen von Brackwasser durch ein meerseitiges Überspülen der Düne (sog. Overtopping). Da das Hauptaugenmerk während der Simulation des Sturmhochwassers auf der binnenseitig überspülten Sohlschwelle und der anschließenden Verteilung von Salzwasser in den Gräben lag, wurden die mit dem Overtopping verbundenen Prozesse der kleinräumigen Salzwasserintrusion über die Düne nicht weiter berücksichtigt. Der Anstieg der EC an dieser Stelle begann erst ca. 20h nach Einsetzen des Sturmhochwassers, was plausibel ist, weil für das Einsetzen des Overtopping ein bestimmter Hochwasserstand der Ostsee erreicht werden muss.

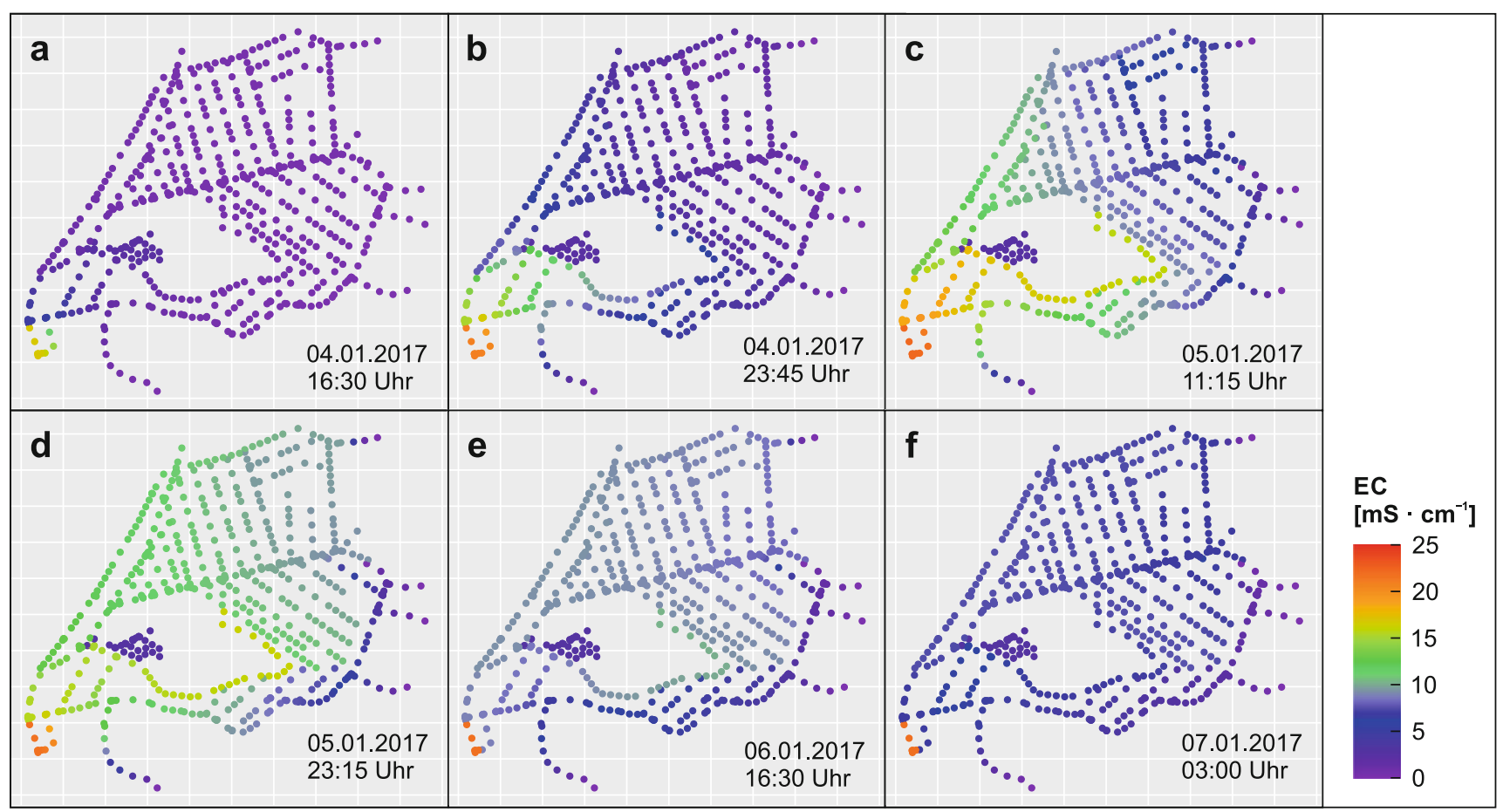

Abb. 6 Simulierte elektr. Leitfähigkeit (EC) in den Gräben des Untersuchungsgebietes während und nach dem Sturmhochwasserereignis

Fig. 6 Spatial distribution of simulated electrical conductivity (EC) in the ditches of the study area during and shortly after the storm flood event 
Die räumlichen Verteilungen der EC im Oberflächenwasser zeigen, dass salzhaltiges Wasser über die Sohlschwelle ins Gebiet eindringt und sich innerhalb eines Tages über die Gräben ausbreitet (Abb. 6a-d). Die räumliche Verteilung der EC in der Niederung ist dabei sehr heterogen (Abb. 6d). Die Konzentration im Waldrandgraben wird stark durch das am Ende des Grabens zuströmende Wasser mit geringen EC beeinflusst, sodass sich die Konzentrationen hier nicht so stark erhöhen wie in den südwestlichen Gräben der Niederung. In den nordöstlichen Bereichen der Niederung (Waldrandgraben) wird das Vordringen des Salzwassers durch das Modell sogar eher noch überschätzt. Bereits kurze Zeit nach dem Ende des Sturmhochwassers zeigt sich eine Aussüßung der Gräben und der Ausgangszustand wird innerhalb von zwei Tagen wieder erreicht (Abb. 6e,f). Die Abnahme der Konzentration erfolgt zum einen durch eine starke Verteilung/Verdünnung in der Fläche und zum anderen durch Nachstrom von Süßwasser über den Waldrandgraben und die Gräben des angrenzenden Waldgebietes.

In der Schilfschicht und im Grundwasser ist kaum ein Anstieg der Salzkonzentrationen, außer in direkter Umgebung der Sohlschwelle zu erkennen. Dies ist als Bestätigung dafür zu werten, dass das schwerere Brackwasser bevorzugt über die Gräben und dort eher sohlnah ins Gebiet vorgedrungen ist und das in den Gräben vorhandene Süßwasser hin zu den Schilfflächen verdrängt hat, wo es zu einer leichten Anhebung des Wasserspiegels gekommen ist. Aus den Gräben wird das eingedrungene Salzwasser innerhalb weniger Tage wieder ausgetragen. Aus diesem Grund kommt es bei dem untersuchten Sturmhochwasser nicht zu einer langanhaltenden Versalzung der Niederung. Lediglich im Nahbereich der Sohlschwelle, in der Salzwasser in den Grundwasserkörper vorgedrungen ist, findet eine anhaltende Versalzung statt.

\section{Schlussfolgerungen}

Das Strömungsverhalten in der Niederung wird geprägt durch den Zustrom aus den Waldgräben sowie dem Abstrom über die Sohlschwelle. Die lateralen oberflächennahen Fließprozesse finden hauptsächlich in den hydraulisch noch aktiven Gräben und der Schilfschicht dazwischen statt. Der zeitlich sehr dynamische unterirdische Austausch mit der Ostsee ist darüber hinaus eine substanzielle und nicht $\mathrm{zu}$ vernachlässigende Komponente der Wasserbilanz der Niederung. Laut den Simulationen erfolgt ein großer Anteil des Grundwasser-Abstroms in die Ostsee durch den Dünensand sowie marinen Sand und nicht durch den Torfkörper unterhalb der Düne. Das Verhalten entlang der Küste wird durch die Dynamik der Ostseewasserstände geprägt. So wechselt sich ein zum Küstengewässer gerichteter Grundwasser-Abstrom mit einem Zustrom von Ostseewasser bei hohen Küs- tenwasserständen innerhalb kurzer Zeiträume ab, wobei der Abstrom im Mittel überwiegt und sich der zeitweise auftretende unterirdische Zufluss aufgrund der hohen Wasserstände in der Niederung nicht weitreichend in das Modellgebiet fortsetzt.

Die Simulation des Sturmhochwasserereignisses zeigt deutlich, dass sich das binnenseitig eindringende salzige Wasser aufgrund der geringeren Strömungswiderstände in den Gräben schneller ausbreitet als im Schilf und im Grundwasser. Aufgrund der Fließdynamik in den Gräben findet keine anhaltende Versalzung der Niederung durch das Sturmhochwasser statt. Speziell das detaillierte Verständnis der vorherrschenden Dynamik als Grundlage für stoffliche Ein- und Austräge ist im Hinblick auf die zukünftige Entwicklung des Küstengebiets von besonderer Bedeutung.

Danksagung DHI-Wasy für die kostenlose Überlassung einer Lizenz für MIKE11-FEFLOW im Rahmen der Erstellung der Masterarbeit von Lisa Schreiber und somit der Grundlage dieses Artikels.

Funding Open Access funding enabled and organized by Projekt DEAL.

Open Access Dieser Artikel wird unter der Creative Commons Namensnennung 4.0 International Lizenz veröffentlicht, welche die Nutzung, Vervielfältigung, Bearbeitung, Verbreitung und Wiedergabe in jeglichem Medium und Format erlaubt, sofern Sie den/die ursprünglichen Autor(en) und die Quelle ordnungsgemäß nennen, einen Link zur Creative Commons Lizenz beifügen und angeben, ob Änderungen vorgenommen wurden.

Die in diesem Artikel enthaltenen Bilder und sonstiges Drittmaterial unterliegen ebenfalls der genannten Creative Commons Lizenz, sofern sich aus der Abbildungslegende nichts anderes ergibt. Sofern das betreffende Material nicht unter der genannten Creative Commons Lizenz steht und die betreffende Handlung nicht nach gesetzlichen Vorschriften erlaubt ist, ist für die oben aufgeführten Weiterverwendungen des Materials die Einwilligung des jeweiligen Rechteinhabers einzuholen.

Weitere Details zur Lizenz entnehmen Sie bitte der Lizenzinformation auf http://creativecommons.org/licenses/by/4.0/deed.de.

\section{Literatur}

Brucher, D.: Verhalten von Chlorid in der ungesättigten Zone und dessen Eignung als hydrologischer Tracer. Diplomarbeit. AlbertLudwigs-Universität, Freiburg (2007). $69 \mathrm{~S}$

Dahms, P.: Studie Wasserregulierung Hütelmoor. unveröffentlicht. Universität Rostock, Fachbereich Landeskultur und Umweltschutz, Fachgebiet Kulturtechnik (1991). 18 S

DHI: A modelling system for rivers and channels: reference manual (2017). https://manuals.mikepoweredbydhi.help/2017/Water Resources/Mike_11_ref.pdf, Zugegriffen: 15. Nov. 2020

DHI: FePEST in FEFLOW 7.0: user guide (2015). https://www. mikepoweredbydhi.com/-/media/shared $\% 20$ content/mike\%20by $\% 20 \mathrm{dhi} /$ flyers\%20and\%20pdf/product-documentation/fepestuser-guide.pdf, Zugegriffen: 15. Nov. 2020

DVWK: Ermittlung der Verdunstung von Land- und Wasserflächen. DVWK-Merkblätter zur Wasserwirtschaft, Bd. 238. Wirtschaftsund Verl.-Ges. Gas und Wasser, Bonn (1996). $135 \mathrm{~S}$

DWD: WESTE-agrar (2018a). https://www.dwd.de/DE/leistungen/ weste/westeagrar/agrar.html, Zugegriffen: 25. Dez. 2018 
DWD: WESTE-XL (2018b). https://www.dwd.de/DE/leistungen/ weste/westexl/weste_xl.html?nn=342632, Zugegriffen: 25. Dez. 2018

Foth, P.: kf-Wert-Messungen nach der Bohrlochmethode im NSG Hütelmoor. unveröffentlicht; MK Rostock, Rostock (1989)

Gelhar, L.W., Welty, C., Rehfeldt, K.R.: A critical review of data on field-scale dispersion in aquifers. Water Resour. Res. 28(7), 1955-1974 (1992). https://doi.org/10.1029/92WR00607

GEOBASIS-DE/M-V: Digitales Geländemodell: Rohdaten. Bearbeitet: Masterarbeit M. Jonas (Hydrologische und geodätische Untersuchungen im NSG „Hütelmoor und Heiligensee“), Universität Rostock (2018)

Hölting, B., Coldewey, W.G.: Hydrogeologie: Einführung in die allgemeine und angewandte Hydrogeologie, 8. Aufl. Springer, Berlin, Heidelberg (2013). $438 \mathrm{~S}$

Ibenthal, M.: Marine and terrestrial influence on submarine groundwater discharge in coastal waters connected to a peatland. Dissertation. Georg-August-Universität Göttingen (2019). http://hdl. handle.net/21.11130/00-1735-0000-0005-13C2-A, $169 \mathrm{~S}$

Jonas, M.: Hydrologische und geodätische Untersuchungen im NSG „Hütelmoor und Heiligensee“. unveröffentlicht; Masterarbeit. Universität Rostock, Rostock (2019)

Jurasinski, G., Janssen, M., Voss, M., Böttcher, M.E., Brede, M., Burchard, H., Forster, S., Gosch, L., Gräwe, U., Gründling-Pfaff, S., Haider, F., Ibenthal, M., Karow, N., Karsten, U., Kreuzburg, M., Lange, X., Leinweber, P., Massmann, G., Ptak, T., Rezanezhad, F., Rehder, G., Romoth, K., Schade, H., Schubert, H., Schulz-Vogt, H., Sokolova, I.M., Strehse, R., Unger, V., Westphal, J., Lennartz, B.: Understanding the Coastal Ecocline: Assessing Sea-Land Interactions at Non-tidal, Low-Lying Coasts Through Interdisciplinary Research. Front. Mar. Sci. 5, Article 342 (2018). https://doi.org/10.3389/fmars.2018.00342

Kreuzburg, M., Ibenthal, M., Janssen, M., Rehder, G., Voss, M., Naumann, M., Feldens, P.: Sub-marine continuation of peat deposits from a coastal Peatland in the southern baltic sea and its holocene development. Front. Earth Sci. 6, Article 103 (2018). https://doi. org/10.3389/feart.2018.00103

Krüger, K.: Untersuchung zur Salzbeeinflussung überflutungsgefährdeter, forstlich genutzter Flächen im Naturschutzgebiet Hütelmoor-Heiligensee. Diplomarbeit. Universität Rostock, Rostock (1995). http://opac.lbs-rostock.gbv.de/DB=1/XMLPRS=N/PPN? PPN=817907556, $83 \mathrm{~S}$

Kulik, D.A., Harff, J.: Physicochemical modeling of the Baltic Sea water-sediment column : I. Reference ion-association models of normative seawater and of Baltic sea brackish waters at salinities $1-40 \%, 1$ bar total pressure and 0 to $30{ }^{\circ} \mathrm{C}$ temperature (system Na-Mg-Ca-K-Sr-Li-Rb-Cl-S-C-Br-F-B-N-Si-P-H-O). Meereswiss. Ber., Bd. 6, Warnemünde (1993). $80 \mathrm{~S}$

LAiV M-V, AfGVK M-V:: GDI-MV - Digitale Orthophotos (DOP) (2018). https://www.geoportal-mv.de/portal/Geowebdienste/Fach themen/Topographische_Karten_und_Luftbilder, Zugegriffen: 22. Aug. 2018

Langevin, C.D., Swain, E.D., Wolfert, M.A.: Simulation of integrated surface-water/ground-water flow and salinity for a coastal wetland and adjacent estuary. J Hydrol. 314(1-4), 212-234 (2005)

Langguth, H.R., Voigt, R.: Hydrogeologische Methoden Bd. 11. Springer, Berlin, Heidelberg, New York (1980). $486 \mathrm{~S}$

LLUR SH: Potentiale und Ziele zum Moor- und Klimaschutz: Gemeinsame Erklärung der Naturschutzbehörden. LLUR SH - Natur, Bd. 20. Pirwitz Druck \& Design, Kiel (2012). 37 S

LUNG M-V: GeoPortal.MV: MV Hydrogeologie WMS (2018). https:// www.geoportal-mv.de/portal/Geowebdienste/Fachthemen/Wasser, Zugegriffen: 20. Juni 2018

Miegel, K., Graeff, T., Selle, B., Salzmann, T., Franck, C., Bronstert, A.: Untersuchung eines renaturierten Niedermoores an der mecklenburgischen Ostseeküste: Teil I: Systembeschreibung und hydrologische Grundcharakterisierung. HyWa 60(4), 242-258 (2016). https://doi.org/10.5675/HyWa_2016,4_1

Miegel, K., Gräff, T., Franck, C., Salzmann, T., Bronstert, A., Walther, M., Oswald, S.E.: Auswirkungen des Sturmhochwassers der Ostsee am 4./5. Januar 2017 auf das renaturierte Niedermoor „Hütelmoor und Heiligensee“ an der deutschen Ostseeküste. HyWa 61(4), 232-243 (2017). https://doi.org/10.5675/HyWa_2017,4_2

Monninkhoff, B.: IfmMIKE11 2.1: Coupling the groundwater model FEFLOW ${ }^{\circledR}$ and the surface water model MIKE ${ }^{\circledR}$. User Manual. DHI-WASY GmbH (2014). https://www.mikepoweredbydhi. com/download/-/media/5d4bdb0c90764ad38e7d161a639e7294. ashx, Zugegriffen: 15. Nov. 2020

Monninkhoff, B., Li, Z.: Coupling FEFLOW and MIKE11 to optimise the flooding system of the lower Havel polders in Germany. Int. J. Water 5(2), 163-180 (2009)

Monninkhoff, B., Altenkirch, N., Luo, J., Clausnitzer, V.: Analyzing the effects of groundwater influenced flooding of a large abandoned mining area in eastern Germany. In: Sui, W., Sun, Y., Wang, C. (Hrsg.) Annual international mine water association conference-an interdisciplinary response to mine water challenges, S. 725-730. China University of Mining and Technology Press, Xuzhou (2014)

OpenStreetMap-Mitwirkende (2020). https://www.openstreetmap.org/ copyright, Zugegriffen: 15. Nov. 2020

Poisson, A., Papaud, A.: Diffusion coefficients of major ions in seawater. Mar. Chem. 13(4), 265-280 (1983)

Qu, W., Li, H., Huang, H., Zheng, C., Wang, C., Wang, X., Zhang, Y.: Seawater-groundwater exchange and nutrients carried by submarine groundwater discharge in different types of wetlands at Jiaozhou Bay, China. J Hydrol. 555, 185-197 (2017). https://doi. org/10.1016/j.jhydrol.2017.10.014

Rechenbach, N.: Experimentelle Bestimmung der Grundwasserströmungsverhältnisse im Hütelmoor. Bachelorarbeit, unveröffentlicht. 67 S. (2009)

Richter, D.: Zur einheitlichen Berechnung der Wassertemperatur und der Verdunstung von freien Wasserflächen auf statistischer Grundlage. Abhandlungen des Meteorologischen Dienstes der Deutschen Demokratischen Republik, Bd. 16, Nr. 119, Akademie-Verlag, Berlin (1977). $35 \mathrm{~S}$

Richter, D.: Ergebnisse methodischer Untersuchungen zur Korrektur des systematischen Messfehlers des Hellmann-Niederschlagsmessers. Selbstverl. des Dt. Wetterdienstes, Offenbach (1995). 93 $\mathrm{S}$

Rossi, P.M., Ala-aho, P., Ronkanen, A.-K., Kløve, B.: Groundwater-surface water interaction between an esker aquifer and a drained fen. J Hydrol. 432-433, 52-60 (2012). https://doi.org/10. 1016/j.jhydrol.2012.02.026

Schreiber, L.: Numerische Modellierung des Fließverhaltens von Grund- und Oberflächenwasser sowie des Stofftransports in einem renaturierten Küstenmoor: Masterarbeit, unveröffentlicht. LIX, Universität Potsdam (2019). $81 \mathrm{~S}$

Selle, B., Graeff, T., Oswald, S.E., Walther, M., Miegel, K.: Untersuchung eines renaturierten Mooreinzugsgebiets an der mecklenburgischen Ostseeküste: Teil II: Salzdynamik und Wasserhaushalt. HyWa 60(4), 259-268 (2016). https://doi.org/10.5675/HyWa 2016,4_2

Thompson, J.R., Refstrup Sørenson, H., Gavin, H., Refsgaard, A.: Application of the coupled MIKE SHE/MIKE 11 modelling system to a lowland wet grassland in southeast England. J Hydrol. 293(1-4), 151-179 (2004)

Umweltministerium M-V: Die Naturschutzgebiete in MecklenburgVorpommern. Demmler, Schwerin (2003). $713 \mathrm{~S}$

WSA Stralsund: Stündliche (für den Zeitraum 01.11.2009 bis 31.05. 2018) und minütliche (für den Zeitraum 01.12.2016 bis 28.02. 2017) Terminwerte des Ostseepegels Warnemünde (2018) 
Yang, J., Graf, T., Herold, M., Ptak, T.: Modelling the effects of tides and storm surges on coastal aquifers using a coupled surfacesubsurface approach. J. Contam. Hydrol. 149, 61-75 (2013)

Hinweis des Verlags Der Verlag bleibt in Hinblick auf geografische Zuordnungen und Gebietsbezeichnungen in veröffentlichten Karten und Institutsadressen neutral. 